\title{
ACTUALIZACIÓN A LA LISTA DE COLEÓPTEROS (INSECTA: COLEOPTERA) DEL BOSQUE DE NEBLINA DE CUYAS, AYABACA-REGIÓN PIURA, PERÚ
}

\author{
Gino Juárez-Noé1,** \& Uzbekia González-Coronado² \\ 1,2 Distrito de Piura, Región de Piura-Perú. Email: norbiol@hotmail.com - ORCID iD: https://orcid.org/0000-0002-8847-6059 \\ ${ }^{2}$ Email: issa.gonzalez06@gmail.com - ORCID iD: https://orcid.org/0000-0001-5920-7549 \\ *Autor para correspondencia: norbiol@hotmail.com
}

\section{RESUMEN}

Con base en colectas y revisión de material depositado en colecciones entomológicas se realiza una actualización a la lista de coleópteros del bosque de neblina de Cuyas, un importante bosque montano de la vertiente occidental de los Andes en la provincia de Ayabaca, región Piura, al noroeste de Perú. Se adicionan 31 especies, 23 géneros, cinco familias y se completa la identificación hasta el nivel de especie de 14 taxones citados en el listado previo, quedando la lista final conformada por 81 especies, 70 géneros, 21 familias y dos subórdenes. La familia Scarabaeidae y los géneros Epilachna Chevrolat, 1837, Astylus Laporte de Castelnau, 1836 y Golofa Hope, 1837 presentan mayor cantidad de especies. Los géneros Dercylus Laporte de Castelnau, 1832, Platynus Bonelli, 1810, Dyscolus Dejean, 1831, Platydracus Thomson, 1858, Belonuchus Bordmann, 1837, Stenocrates Burmeister, 1847 y Mycotretus Lacordaire, 1842 son nuevos registros para la región Piura. Todas las especies consideradas en esta actualización se presentan a modo de listado taxonómico comentado incluyendo para cada uno datos de colecta.

Palabras clave: Coleoptera, actualización, distribución, bosques montanos, Ayabaca, Piura.

\section{ABSTRACT}

Update of the checklist of beetles (Insecta: Coleoptera) of Cuyas cloud forest, Ayabaca-Piura region, Peru

We update the checklist of beetles from the Cuyas cloud forest, an important montane forest on the western slope of the Andes in Ayabaca province, Piura region, northwest of Peru based on specimen collecting and a review of the material deposited in entomological collections. 31 species, 23 genera, five families are added and we complete the identification to species level for 14 genera in the previous checklist, increasing the current taxonomic diversity to 81 species, 70 genera, 21 families and two suborders. Family Scarabaeidae and genera Epilachna Chevrolat, 1837, Astylus Laporte de Castelnau, 1836 and Golofa Hope, 1837 are the most species-rich. Genera Dercylus Laporte de Castelnau, 1832, Platynus Bonelli, 1810, Dyscolus Dejean, Platydracus Thomson, 1858, Belonuchus Bordmann, 1837, Stenocrates Burmeister, 1847 and Mycotretus Lacordaire, 1842 are new records for the Piura region. The species considered in this update are presented as a commented taxonomic list including collection data for each species.

Keywords: Coleoptera, update, distribution, montane forests, Ayabaca, Piura.

Recibido/Received: 24/04/2020; Aceptado/Accepted: 20/10/2020; Publicado en línea/Published online: 11/05/2021

Cómo citar este artículo/Citation: Juárez-Noé, G. \& González-Coronado, U. 2021. Actualización a la lista de coleópteros (Insecta: Coleoptera) del bosque de neblina de Cuyas, Ayabaca-Región Piura, Perú. Grael/sia, 77(1): e126. https://doi. org/10.3989/graellsia.2021.v77.278

Copyright: (c) 2021 SAM \& CSIC. Este es un artículo de acceso abierto distribuido bajo los términos de una licencia de uso y distribución Creative Commons Reconocimiento 4.0 Internacional (CC BY 4.0). 


\section{Introducción}

En Perú el orden Coleoptera está representado por más de 10.000 especies descritas y más de 99 familias, siendo uno de los grupos de insectos más diversos del país (Chaboo, 2015). La región Piura cuenta con 317 especies distribuidas en 255 géneros, 38 familias y 2 subórdenes (Juárez-Noé \& González-Coronado, 2018, 2019). Los coleópteros por su gran abundancia, diversidad ecológica y por estar asociados con formaciones vegetales donde actúan como depredadores, herbívoros, polinizadores y descomponedores de materia orgánica, cobran especial importancia en estudios ecológicos, especialmente como indicadores de biodiversidad y conservación (Lozada et al., 2004).

En el norte de Perú, los bosques de neblina, que son relictos de bosque montano de la vertiente occidental de los Andes, se encuentran distribuidos en torno a la zona de deflexión de Huancabamba abarcando las regiones de Piura, Lambayeque, Cajamarca y La Libertad (MINAM, 2018). En Piura, estos bosques se encuentran ubicados en las provincias de Ayabaca, Huancabamba y Morropón, entre los $2.000 \mathrm{~m}$ a $3.000 \mathrm{~m}$ de altitud, siendo el bosque de neblina de Cuyas uno de los más importantes. Este bosque ubicado cerca de la ciudad de Ayabaca, está conformada por bosque primario (en estado natural) y bosque secundario (alterado o en regeneración) (Flanagan \& Vellinga, 2000), dentro de los cuales se encuentran enmarcadas varias comunidades campesinas (Cuchayo, Joras y Suyupampa) (Ambulay, 2006).

El bosque de neblina de Cuyas alberga uno de los principales relictos de bosque montano de la vertiente occidental de los Andes (Fig. 2) y debido a la presencia de especies endémicas de flora y fauna es considerada como área de importancia para la conservación y como sitio prioritario del plan director del sistema nacional de áreas naturales protegidas por el estado en Perú (More et al., 2014).

En este bosque, Juárez \& González (2016a) elaboraron un listado de coleópteros en el que registraron 50 especies, 45 géneros y 16 familias, siendo hasta el momento la única lista que detalla el número de especies del orden Coleoptera presentes para dicho bosque. Por ello, en la presente investigación con la adición de 31 especies, 23 géneros, cinco familias y además con la completa identificación hasta el nivel de especie de 14 taxones citados en el listado previo, actualizamos el número de especies de coleópteros presentes en el bosque de neblina de Cuyas.

\section{Material y métodos}

\section{ÁREA DE ESTUDIO}

El bosque de neblina de Cuyas (43의 $\mathrm{S}, 7^{\circ} 42^{\prime} \mathrm{O}$ y $4^{\circ} 35^{\prime} \mathrm{S}, 79^{\circ} 41^{\prime} \mathrm{O}$ ) está ubicado en el distrito y pro- vincia de Ayabaca, en la región Piura (Fig. 1), específicamente entre las laderas de los cerros Chacas, Yantuma y Campanario en un rango altitudinal desde los $2.000 \mathrm{msnm}$ hasta los casi $3.000 \mathrm{msnm}$. Su clima es templado frío con inviernos secos, temperaturas de $8^{\circ} \mathrm{C}$ a $15^{\circ} \mathrm{C}$, nivel de precipitación de $1.750 \mathrm{~mm}$ a $2.000 \mathrm{~mm}$ al año y humedad del $100 \%$ durante la temporada de lluvias (noviembre-mayo). La presencia de niebla es ligeramente superior a la mitad de un día en la estación seca y más alta en la temporada de lluvias, donde las nubes pueden cubrir el bosque durante largos períodos (Ledo, 2012).

La vegetación predominante está compuesta principalmente por especies de los géneros Meliosma Blume (Sabiaceae), Oreopanax Decne. \& Planch. (Araliaceae), Persea Mill. y Ocotea Aubl. (Lauraceae), Delostoma D. Don (Bignoniaceae), Ruagea H. Karst (Meliaceae), Morus L. (Moraceae), Citronella D. Don (Icacinaceae), Myrcianthes O.Berg y Eugenia P. Micheli ex L. (Myrtaceae), Cestrum L. e Iochroma Benth. (Solanaceae), Miconia Ruiz \& Pav. (Melastomataceae) y Parathesis (A.DC.) Hook.f. (Myrsinaceae) entre la vegetación arbórea; Baccharis L. (Asteraceae), Piper L. (Piperaceae), Solanum L. (Solanaceae), Verbesina L. (Asteraceae) y Fuchsia L. (Onagraceae) entre los arbustos, mientras que el género Chusquea Kunth (Poaceae) destaca entre la vegetación herbácea, además de abundantes musgos, líquenes y orquídeas (Farfán, 2007; Ledo, 2012).

\section{OBtención de datos}

El bosque fue dividido en tres áreas de muestreo de acuerdo a su altitud (msnm), siguiendo los criterios propuestos por Juárez \& González (2016a) en el listado previo: estrato bajo desde los 2.000 a $2.300 \mathrm{msnm}$ (zona de Ambasal), estrato medio desde los 2.300 a 2.600 msnm (zona La Cruz y zona Bernando-Calle) y estrato alto desde los 2.600 hasta aproximadamente $3.000 \mathrm{msnm}$ (zona montaña abierta).

En cada área se utilizaron 4 técnicas de muestreo: (1) trampas pitfall, formadas por vasos plásticos transparentes de $500 \mathrm{ml}$ y $9 \mathrm{~cm}$ de diámetro de abertura llenados hasta su mitad con una mezcla de siete partes de agua por una de formol al $40 \%$ y un poco de detergente, cebadas con pescado podrido y plátano fermentado con miel de caña y cerveza negra (Giraldo \& Arellano, 2003; Saavedra, 2010) e instaladas en un transecto lineal de $100 \mathrm{~m}$ con 10 trampas separadas cada $10 \mathrm{~m}$ y revisadas cada 12 horas (Villarreal et al., 2004). (2) Una trampa luz formada por dos focos de 100 vatios cada uno de luz blanca colocadas frente y debajo de mantas blancas de $2 \times 2 \mathrm{~m}$, las cuales estuvieron activas entre las 19:00 h y 23:00 h (Solís, 2004). (3) En pequeñas quebradas y cuerpos de agua de aproximadamente $1.50 \mathrm{~m}$ de profundidad se utilizó una red acuática de $30 \mathrm{~cm}$ de diámetro, $40 \mathrm{~cm}$ de fondo, con una malla de $0,1 \mathrm{~mm}$ (Benetti et al., 2003) 


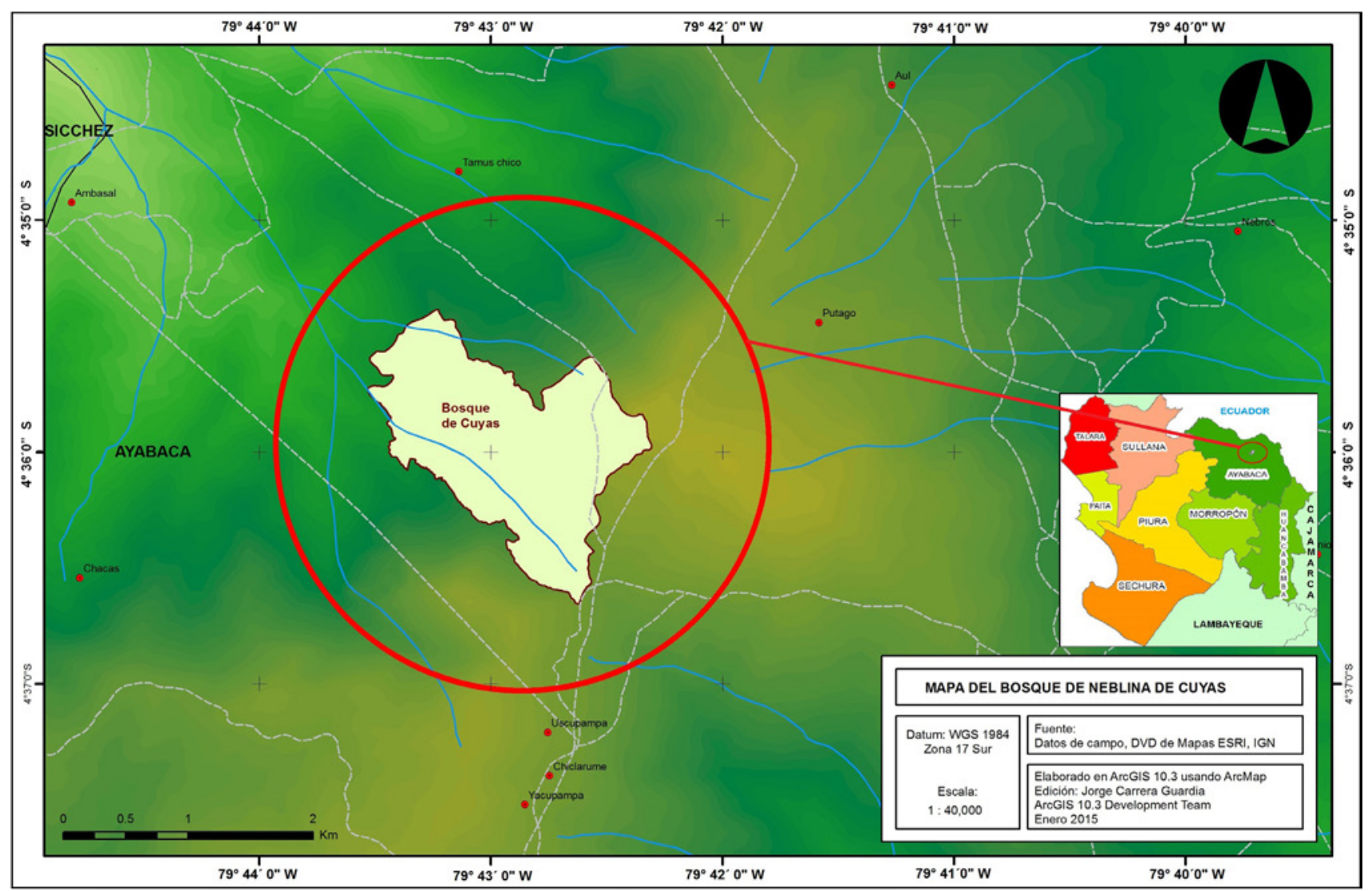

Fig. 1.- Ubicación del bosque de neblina de Cuyas (fuente: Palacios-Noé, 2016).

Fig. 1.- Location of the Cuyas cloud forest (source: Palacios-Noé, 2016).

adherida a una vara rígida de un metro de largo. (4) Por último, se realizó recolecta directa, tanto diurna como nocturna, revisando el follaje de las plantas, flores, hojarasca, troncos podridos, bajo piedras, animales en descomposición y excremento (Morón, 2004).

Las colectas tuvieron una duración de 2 días por área y se llevaron a cabo durante los meses de enero, marzo, junio, julio, octubre y noviembre del 2018 y febrero, abril, junio, agosto, setiembre y diciembre del 2019.

Adicionalmente, se revisó material en la colección Gino Juárez Noé (GJN) Piura-Perú, donde se encuentran depositados los ejemplares citados por Juárez \& González (2016a) en la lista inicial de coleópteros para el bosque de Cuyas, la cual permitió completar la identificación hasta el nivel de especie de 14 taxones citados previamente.

Para la identificación de los especímenes colectados y de los ejemplares depositados en la colección GJN se utilizaron los trabajos de Dillon \& Dillon (1941), Balthasar (1942), Bachmann (1966), Ratcliffe (1976), Breuning (1966), Gordon (1975), Scherer (1983), Endrödi (1985), Lachaume (1985), Génier (1996), Pinto \& Bologna (1999), Medina \& LoperaToro (2000), Navarrete et al. (2002), Génier \& Kohlmann (2003), Morón (2003), Smith (2003), Háva (2004), Bartolozzi \& Onore (2006), González (2007),
Smith \& Skelley (2007), Wells (2007), Soula (2002, 2008), Abadie et al. (2008), Ciro-Díaz et al. (2008), Gasca et al. (2008), Archangelsky et al. (2009), Bocákova (2010), Paulsen (2010), Constantin (2011), Libonatti et al. (2011), Onore et al. (2011), Vaz de Mello et al. (2011), Beenen (2013), Santos-Silva et al. (2013), Solís \& Kohlmann (2013), Martins \& Galileo (2014), Shaughney \& Ratcliffe (2015), Blanco-Aller (2016), Figueroa \& Ratcliffe (2016), Miller \& Bergsten (2016), Noguchi \& Santos-Silva (2016), Bustamante-Navarrete et al. (2017) y Ratcliffe \& Le Tirant (2017). Los ejemplares estudiados en esta actualización fueron depositados en la colección del Museo de Entomología de la Universidad Nacional de Tumbes, Perú (MEUNT) y en la colección privada Gino Juárez Noé (CGJN) en Piura, Perú.

La información obtenida se presenta a modo de listado taxonómico (orden, familia, subfamilia, género y especie) y se incluyen comentarios sobre cada taxón. El listado se ordena siguiendo la clasificación de Bouchard et al. (2011), sin embargo, dentro de Chrysomelidae se considera a Alticinae como subfamilia (Furth \& Suzuki, 1998; Mohamedsaid \& Furth, 2011; Furth et al., 2015). La secuencia del material estudiado fue la siguiente: número de $\hat{\delta} \hat{\partial}$, $ᄋ \underline{\text {, }}$, provincia, distrito, zona de muestreo, coordenadas geográficas, altitud en msnm, fecha de captura, tipo de captura, colector y 


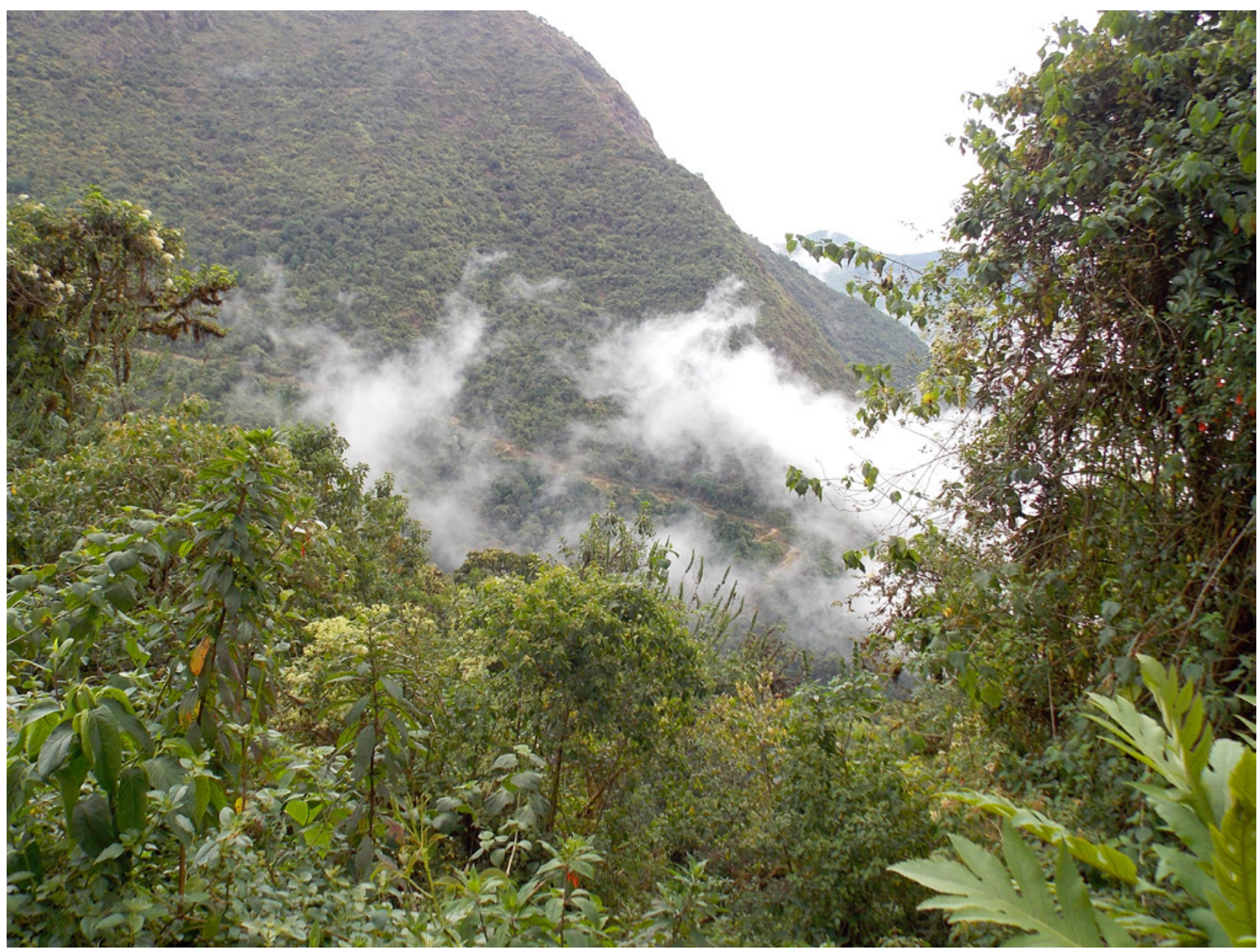

Fig. 2.- Vista del bosque de neblina de Cuyas.

Fig. 2.- View of Cuyas cloud forest.

código de registro cuando procedió de material depositado en la colección GJN. Los taxones citados por primera vez en la lista se indican con una cruz $(+)$, mientras que las especies conocidas únicamente de Perú (Bartolozzi \& Onore, 2006; Ratcliffe et al., 2015; Noguchi \& Santos-Silva, 2016; Ratcliffe \& Le Tirant, 2017; Juárez-Noé \& González-Coronado, 2020), se indican con un asterisco $\left(^{*}\right)$.

\section{Resultados y discusión}

En total se obtuvieron 309 ejemplares a través de los diferentes métodos de colecta. A partir de estos 309 ejemplares, 5 familias, 23 géneros y 31 especies no estaban registradas en la lista inicial de coleópteros para el bosque de neblina de Cuyas (Juárez \& González, 2016a). La revisión del material identificado en el estudio de Juárez \& González (2016a), permitió asignar el epíteto específico a 14 taxones: Sphaenognathus Buquet, 1838, Platycoelia Dejean, 1833, Strigidia Dejean, 1833, Pseudothyridium Soula, 2002, Megaceras Hope, 1837, Heterogomphus Burmeister, 1847, Ho- plopyga Thomson, 1880, Semiotus Eschscholtz, 1829, Psalidognathus Gray, 1831, Bisaltes Thomson, 1868, Fredlanea Martins \& Galileo, 1996, Diabrotica Chevrolat, 1837, Sceloenopla Chevrolat, 1837 y Compsus Schoenherr, 1823

En la lista de Juárez \& González (2016a) de los coleópteros del bosque de neblina de Cuyas se registraron 33 especies pertenecientes a 45 géneros y 16 familias de coleópteros, adicionalmente se reportaron 17 taxones a los que no se pudo determinar el epíteto específico que acompaña al nombre genérico los cuales se designaron como sp. Por lo tanto, con la adición de las 31 especies, 23 géneros y cinco familias provenientes de colectas y de la asignación del epíteto específico a los 14 taxones identificados por Juárez \& González (2016a), la nueva lista de coleópteros para el bosque de neblina de Cuyas queda conformada por 21 familias, 70 géneros y 81 especies, 59 acompañadas con su epíteto específico y 22 designadas como sp.

Las familias añadidas a la lista son Gyrinidae, Dytiscidae, Hydrophilidae, Silphidae y Erotylidae. El total de familias registradas representan el $21 \%$ de las citadas a nivel nacional (Chaboo, 2015) y el 
$55 \%$ de las citadas a nivel regional (Juárez-Noé \& González-Coronado, 2019), siendo Scarabaeidae con 31, Chrysomelidae con 7, Dytiscidae, Coccinellidae con 6 cada una y Cerambycidae con 5 las que presentan mayor cantidad de especies, destacando asimismo los géneros Epilachna Chevrolat, 1837 con 4 especies, Golofa Hope, 1837 y Astylus Laporte de Castelnau, 1836 con 3 especies cada una. Las siguientes especies son conocidas únicamente en Perú: Ontherus rectus Génier, 1996, Thyriochlorota villosa (Ohaus, 1908), Sphaenognathus (Chasiognathinus) xerophilus Bartolozzi \& Onore, 2006, Golofa limogesi Ratcliffe \& Le Tirant, 2017, Psalidognathus antonkoslovi Noguchi \& Santos-Silva, 2016 y Fredlanea wilderi Juárez-Noé \& González-Coronado, 2020 (Bartolozzi \& Onore, 2006; Ratcliffe et al., 2015; Noguchi \& Santos-Silva, 2016; Ratcliffe \& Le Tirant, 2017; JuárezNoé \& González-Coronado, 2020).

La cantidad de especies registradas en la presente actualización es muy superior si se toma como referencia a las 35 especies de coleópteros reportadas por Saavedra et al. (2017) para los bosques de Ramos y Chin-Chin, bosques de neblina ubicados en las provincias de Ayabaca y Huancabamba respectivamente. Del mismo modo, las 81 especies de coleópteros registrados aquí junto con las 46 especies de lepidópteros diurnos reportadas por Zelada (2004) para el mismo bosque, hace entender que el bosque de neblina de Cuyas posee una variada riqueza insectil.

Es importante mencionar que las colectas de algunas especies resultaron ser nuevos registros para la región Piura (Juárez-Noé \& González-Coronado, 2019), tal es el caso de los géneros Dercylus Laporte de Castelnau, 1832, Platynus Bonelli, 1810, Dyscolus Dejean, 1831, Platydracus Thomson, 1858, Belonuchus Bordmann, 1837, Stenocrates Burmeister, 1847 y Mycotretus Lacordaire, 1842. Estos registros demuestran la importancia de las colectas entomológicas en áreas con gran biodiversidad faunística (More et al., 2014).

Por otro lado, el $90 \%$ de las especies registradas en la presente actualización muestran concordancia con el tipo de paisaje ecológico en el cual se encuentra inmerso el bosque de neblina de Cuyas (More et al., 2014; MINAM, 2018), mientras que el $10 \%$ de especies restantes muestran amplia distribución, abarcando todos los paisajes ecológicos de la región Piura, esto específicamente para Megadytes (Bifurcitus) Ihermineri (Guérin-Méneville, 1829), Meridiorhantus calidus (Fabricius, 1792), Thermonectus succinctus (Aubé, 1838), Tropisternus (Strepitornus) collaris (Fabricius, 1775), Hydrophilus (Hydrophilus) foveolatus (Régimbart, 1901), Mecopelidnota marxi Soula, 2008, Paranomala undulata peruviana GuérinMéneville, 1831, Aphodius (Labarrus) pseudolividus Balthasar, 1941 y Attagenus (Attagenus) fasciatus (Thunberg, 1795) que se adaptan tanto a zonas secas y desérticas como a bosques estacionalmente secos y desiertos costeros (Juárez-Noé \& González-Coronado, 2018, 2019).

E1 $84 \%$ de las especies (42 especies) citadas por Juárez \& González (2016a) en la lista inicial fueron recolectadas nuevamente lo que permitió confirmar las mismas, mientras que no se lograron recolectar los siguientes taxones previamente citados: Dyscolus sin identificar sp 1, Platynus sin identificar sp 1, Platydracus sin identificar sp 1, Scatimus monstrosus Balthasar, 1939, Uroxys sin identificar sp 1, Onthophagus sin identificar sp 1, Psilodon sin identificar sp 1 y Semiotus $\sin$ identificar sp 1 . Del total de insectos registrados en la presente actualización el $72 \%$ se identificó hasta el nivel de especie y el $28 \%$ hasta el nivel de género. Estos porcentajes difieren con el nivel de identificación respecto al trabajo anterior, ya que solo el $36 \%$ de los insectos registrados se identificó hasta especie (Juárez \& González, 2016a).

En cuanto a los métodos de captura, 51 especies (63\% de las colectadas) se recogieron a través de colecta manual, siendo 44 de ellas exclusivas de este método, mientras que el $37 \%$ restante se recogieron a través de trampas pitfall (ocho especies, todas exclusivas), trampas luz (quince especies, 10 de ellas exclusivas) y con red acuática (cinco especies, todas exclusivas). Mecopelidnota marxi, Megaceras morpheus Burmeister, 1847, A. pseudolividus y las especies de Golofa se capturaron tanto con trampa luz como por colecta manual.

Por último, es necesario seguir realizando colectas de forma continuada especialmente en áreas poco exploradas dentro del bosque, por lo que probablemente la diversidad de coleópteros del bosque de neblina de Cuyas se encuentre aún subestimada.

LisTA TAXONÓMICA

Suborden Adephaga Schellenberg, 1806

Familia Gyrinidae Latreille, $1810+$

Subfamilia Gyrininae Latreille, $1810+$

Género Andorygus Ochs, $1924+$

1. Andorygus peruvianus Régimbart, $1907+$

Material examinado. $1 \hat{\jmath}$, Piura, Ayabaca, Bosque de neblina de Cuyas, estrato alto, $05^{\circ} 10^{\prime} 51^{\prime \prime} \mathrm{S}-80^{\circ} 37^{\prime} 31^{\prime \prime} \mathrm{O}, 2,834 \mathrm{msnm}, 15-$ VI-2018, red acuática, G. Juárez.

Comentario. Especie característica de cuerpos de agua de alta montaña en los Andes. En Perú se cita también para la región Cusco (Bustamante, 2018).

Familia Carabidae Latreille, 1802

Subfamilia Harpalinae Bonelli, 1810

Género Dercylus Laporte de Castelnau, 1832

2. Dercylus sin identificar sp. 1.

Material examinado. $1 \hat{\jmath}$, Piura, Ayabaca, Bosque de neblina de Cuyas, estrato medio, $05^{\circ} 10^{\prime} 51^{\prime \prime} \mathrm{S}-80^{\circ} 37^{\prime} 31^{\prime \prime} \mathrm{O}, 2,597 \mathrm{msnm}$, 15-VI-2018, trampas pitfall, G. Juárez.

Comentario. El género Dercylus se distribuye en bosques montanos y bosques de la cuenca amazónica de Perú, Venezuela, Colombia, Ecuador, Brasil, Guyana y Bolivia (Moret \& Bousquet, 1995; Moret, 2005). Nuevo registro del género para la región Piura (Juárez-Noé \& González-Coronado, 2019). 
Género Platynus Bonelli, 1810

3. Platynus sin identificar sp. 1.

Material examinado. 1 , Piura, Ayabaca, Bosque de neblina de Cuyas, estrato medio, $05^{\circ} 10^{\prime} 51^{\prime \prime} \mathrm{S}-80^{\circ} 37^{\prime} 31^{\prime \prime} \mathrm{O}, 2,493 \mathrm{msnm}$, 23-III-2016, trampas pitfall, G. Juárez.

Comentario. Nuevo registro del género para la región Piura (Juárez-Noé \& González-Coronado, 2019).

Género Dyscolus Dejean, 1831

4. Dyscolus sin identificar sp. 1.

Material examinado. $1 \hat{\delta}$, Piura, Ayabaca, Bosque de neblina de Cuyas, estrato medio, $05^{\circ} 10^{\prime} 51^{\prime \prime} \mathrm{S}-80^{\circ} 37^{\prime} 31^{\prime \prime} \mathrm{O}, 2,475 \mathrm{msnm}$, 15-VI-2016, trampas pitfall, G. Juárez.

Comentario. El género Dyscolus se distribuye en zonas de alta montaña de América Central, El Caribe y en zonas de bosques montanos y páramos de Colombia, Bolivia, Ecuador, Argentina, Chile y Perú (Moret, 2005). Nuevo registro del género para la región Piura (Juárez-Noé \& González-Coronado, 2019).

Familia Dytiscidae Leach, $1815+$

Subfamilia Agabinae Thomson, 1867

Género Agametrus Sharp, $1882+$

5. Agametrus andinus Guignot, $1958+$

Material examinado. 1 $\hat{\delta}$, Piura, Ayabaca, Bosque de neblina de Cuyas, estrato medio, $05^{\circ} 10^{\prime} 51^{\prime \prime} \mathrm{S}-80^{\circ} 37^{\prime} 31^{\prime \prime} \mathrm{O}, 2,475 \mathrm{msnm}, 10$ VIII-2019, red acuática, G. Juárez; 1 §., Piura, Ayabaca, Bosque de neblina de Cuyas, estrato alto, $05^{\circ} 10^{\prime} 51^{\prime \prime} \mathrm{S}-80^{\circ} 37^{\prime} 31^{\prime \prime} \mathrm{O}$, 2,743 msnm, 03-XII-2019, red acuática, G. Juárez.

Comentario. Las especies del género Agametrus son característicos de cuerpos de agua de alta montaña (Miller \& Bergsten, 2016). En la región Piura $A$. andinus se distribuye en zonas de bosque montano de la provincia de Ayabaca (Juárez-Noé \& GonzálezCoronado, 2019).

Subfamilia Copelatinae Branden, 1885

Género Copelatus Erichson, $1832+$

6. Copelatus blancasi Guignot, $1958+$

Material examinado. $1 \hat{\jmath}$, Piura, Ayabaca, Bosque de neblina de Cuyas, estrato medio, $05^{\circ} 10^{\prime} 51^{\prime \prime} \mathrm{S}-80^{\circ} 37^{\prime} 31^{\prime \prime} \mathrm{O}, 2,400 \mathrm{msnm}$, 11-II-2019, red acuática, G. Juárez; 1 ô, Piura, Ayabaca, Bosque de neblina de Cuyas, estrato medio, $5^{\circ} 10^{\prime} 51^{\prime \prime} \mathrm{S}-80^{\circ} 37^{\prime} 31^{\prime \prime} \mathrm{O}$, 2,387 msnm, 13-IV-2019, red acuática, G. Juárez.

Comentario. En la región Piura se distribuye en zonas de bosque montano de la provincia de Ayabaca (Juárez-Noé \& GonzálezCoronado, 2019).

Subfamilia Laccophilinae Gistel, 1856

Género Laccophilus Leach, $1815+$

7. Laccophilus normifer Guignot, $1958+$

Material examinado. $1 \hat{\delta}$, Piura, Ayabaca, Bosque de neblina de Cuyas, estrato medio, $05^{\circ} 10^{\prime} 51^{\prime \prime} \mathrm{S}-80^{\circ} 37^{\prime} 31^{\prime \prime} \mathrm{O}, 2,400 \mathrm{msnm}$, 23-VI-2019, red acuática, G. Juárez.

Comentario. En la región Piura se distribuye en zonas de bosque montano de la provincia de Ayabaca (Juárez-Noé \& GonzálezCoronado, 2019).

Subfamilia Dytiscinae Leach, 1815

Género Megadytes Sharp, $1882+$

8. Megadytes (Bifurcitus) Ihermineri (Guérin-Méneville, 1829) +

Material examinado. $2 \hat{\jmath} \widehat{\jmath}$, Piura, Ayabaca, Bosque de neblina de Cuyas, estrato bajo, $05^{\circ} 10^{\prime} 51^{\prime \prime} \mathrm{S}-80^{\circ} 37^{\prime} 31^{\prime \prime} \mathrm{O}, 2,112 \mathrm{msnm}$, 28-I-2018, trampa luz, G. Juárez; 1 ô, Piura, Ayabaca, Bosque de neblina de Cuyas, estrato medio, $5^{\circ} 10^{\prime} 51^{\prime \prime} \mathrm{S}-80^{\circ} 37^{\prime} 31^{\prime \prime} \mathrm{O}$, 2,534 msnm, 09-VII-2018, trampa luz, G. Juárez; 1 ô, Piura, Ayabaca, Bosque de neblina de Cuyas, estrato medio, $05^{\circ} 10^{\prime} 51^{\prime \prime} \mathrm{S}-80^{\circ} 37^{\prime} 31^{\prime \prime} \mathrm{O}, 2,412 \mathrm{msnm}, 27-\mathrm{IV}-2019$, trampa luz,
U. González; 1 ^̂, Piura, Ayabaca, Bosque de neblina de Cuyas, estrato alto, $5^{\circ} 10^{\prime} 51^{\prime \prime} \mathrm{S}-80^{\circ} 37^{\prime} 31^{\prime \prime} \mathrm{O}, 2,834 \mathrm{msnm}, 06-\mathrm{XI}-2018$, trampa luz, G. Juárez.

Comentario. Especie registrada en Bahamas, Cuba, Haití, Guatemala, Puerto Rico, México, Brasil, Venezuela y Perú (Blanco-Aller, 2016; Juárez-Noé \& González-Coronado, 2018). La especie es de amplia distribución en la región Piura abarcando todas las provincias y paisajes ecológicos (JuárezNoé \& González-Coronado, 2018, 2019).

Género Thermonectus Dejean, $1837+$

9. Thermonectus succinctus (Aubé, 1838) +

Material examinado. 1 $\widehat{\text { }}$, Piura, Ayabaca, Bosque de neblina de Cuyas, estrato bajo, $05^{\circ} 10^{\prime} 51^{\prime \prime} \mathrm{S}-80^{\circ} 37^{\prime} 31^{\prime \prime} \mathrm{O}, 2,034 \mathrm{msnm}$, 28-I2018, trampa luz, U. González; 1 đै, Piura, Ayabaca, Bosque de neblina de Cuyas, estrato medio, 510'51' S-80 $37^{\prime} 31^{\prime \prime} \mathrm{O}, 2,312$ msnm, 10-VII-2018, trampa luz, G. Juárez; 1 đૈ, Piura, Ayabaca, Bosque de neblina de Cuyas, estrato alto, $5^{\circ} 10^{\prime} 51^{\prime \prime} \mathrm{S}-80^{\circ} 37^{\prime} 31^{\prime \prime} \mathrm{O}$, 2,721 msnm, 26-II-2019, trampa luz, G. Juárez.

Comentario. Especie que se distribuye en Costa Rica, Cuba, México, Brasil, Argentina, Bolivia, Colombia, Paraguay, Uruguay y Perú (Blanco-Aller, 2016). Es de amplia distribución en la región Piura abarcando todas las provincias y paisajes ecológicos (Juárez-Noé \& González-Coronado, 2018, 2019).

Subfamilia Colymbetinae Erichson, 1837

Género Meridiorhantus Balke, Hájek \& Hendrich, 2017 +

10. Meridiorhantus calidus (Fabricius, 1792) +

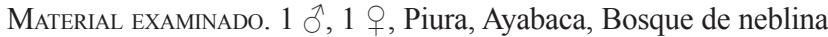
de Cuyas, estrato bajo, $05^{\circ} 10^{\prime} 51^{\prime \prime} \mathrm{S}-80^{\circ} 37^{\prime} 31^{\prime \prime} \mathrm{O}, 2,003 \mathrm{msnm}, 04-$ IX-2019, trampa luz, G. Juárez; 1 , Piura, Ayabaca, Bosque de neblina de Cuyas, estrato medio, $5^{\circ} 10^{\prime} 51^{\prime \prime} \mathrm{S}-80^{\circ} 37^{\prime} 31^{\prime \prime} \mathrm{O}, 2,435$ msnm, 10-II-2019, trampa luz, G. Juárez; 1 §ె, Piura, Ayabaca, Bosque de neblina de Cuyas, estrato alto, $5^{\circ} 10^{\prime} 51^{\prime \prime} \mathrm{S}-80^{\circ} 37^{\prime} 31^{\prime \prime} \mathrm{O}$, 2,819 msnm, 13-VI-2019, trampa luz, U. González.

Comentario. Especie de amplia distribución en la región Piura abarcando todas las provincias y paisajes ecológicos (JuárezNoé \& González-Coronado, 2018, 2019).

Suborden Polyphaga Emery, 1886

Familia Hydrophilidae Latreille, $1802+$

Subfamilia Hydrophilinae Latreille, 1802

Género Tropisternus Solier, $1834+$

11. Tropisternus (Strepitornus) collaris (Fabricius, 1775) +

Material examinado. 1 o, Piura, Ayabaca, Bosque de neblina de Cuyas, estrato bajo, $05^{\circ} 10^{\prime} 51^{\prime \prime} \mathrm{S}-80^{\circ} 37^{\prime} 31^{\prime \prime} \mathrm{O}, 2,187 \mathrm{msnm}, 14-\mathrm{X}$ 2018, red acuática, U. González; 2 우, Piura, Ayabaca, Bosque de neblina de Cuyas, estrato medio, $5^{\circ} 10^{\prime} 51^{\prime \prime} \mathrm{S}-80^{\circ} 37^{\prime} 31^{\prime \prime} \mathrm{O}$, 2,534 msnm, 28-VII-2018, red acuática, G. Juárez; 1 ก, Piura, Ayabaca, Bosque de neblina de Cuyas, estrato medio, $5^{\circ} 10^{\prime} 51^{\prime \prime} \mathrm{S}-80^{\circ} 37^{\prime} 31^{\prime \prime} \mathrm{O}, 2,419 \mathrm{msnm}, 29-\mathrm{VII}-2018$, trampa luz, G. Juárez; 1 $\widehat{O}, 1$ \&, Piura, Ayabaca, Bosque de neblina de Cuyas, estrato alto, $05^{\circ} 10^{\prime} 51^{\prime \prime} \mathrm{S}-80^{\circ} 37^{\prime} 31^{\prime \prime} \mathrm{O}, 2,767 \mathrm{msnm}, 04-\mathrm{II}-$ 2019, red acuática, U. González; 1 \&, Piura, Ayabaca, Bosque de neblina de Cuyas, estrato alto, $5^{\circ} 10^{\prime} 51^{\prime \prime} \mathrm{S}-80^{\circ} 37^{\prime} 31^{\prime \prime} \mathrm{O}$, 2,787 msnm, 23-IX-2019, red acuática, G. Juárez.

Comentario. Especie de amplia distribución en América, desde el norte de Canadá hasta el extremo sur de Sudamérica. En la región Piura tiene una amplia distribución abarcando desde bosques estacionalmente secos hasta bosques húmedos de montaña (Juárez-Noé \& González-Coronado, 2018, 2019).

Género Hydrophilus Geoffroy, $1762+$

12. Hydrophilus (Hydrophilus) foveolatus (Régimbart, 1901) +

Material examinado. 1 , Piura, Ayabaca, Bosque de neblina de Cuyas, estrato bajo, $05^{\circ} 10^{\prime} 51^{\prime \prime} \mathrm{S}-80^{\circ} 37^{\prime} 31^{\prime \prime} \mathrm{O}, 2,199 \mathrm{msnm}, 02-$ XI-2018, trampa luz, U. González; 1 ㅇ, Piura, Ayabaca, Bosque 
de neblina de Cuyas, estrato medio, $5^{\circ} 10^{\prime} 51^{\prime \prime} \mathrm{S}-80^{\circ} 37^{\prime} 31^{\prime \prime} \mathrm{O}$, 2,312 msnm, 08-III-2018, trampa luz, G. Juárez; 1 ô, 1 q, Piura, Ayabaca, Bosque de neblina de Cuyas, estrato alto, $05^{\circ} 10^{\prime} 51^{\prime \prime} \mathrm{S}-80^{\circ} 37^{\prime} 31^{\prime \prime} \mathrm{O}, 2,832 \mathrm{msnm}, 01-\mathrm{IV}-2019$, trampa luz, G. Juárez; 1 + , Piura, Ayabaca, Bosque de neblina de Cuyas, estrato alto, $5^{\circ} 10^{\prime} 51^{\prime \prime} \mathrm{S}-80^{\circ} 37^{\prime} 31^{\prime \prime} \mathrm{O}, 2,621 \mathrm{msnm}, 13-\mathrm{IX}-2019$, trampa luz, G. Juárez.

Comentario. Especie de amplia distribución en la región Piura abarcando todas las provincias y paisajes ecológicos (JuárezNoé \& González-Coronado, 2018, 2019).

Familia Silphidae Latreille, $1806+$

Subfamilia Silphinae Latreille, 1806

Género Oxelytrum Gistel, $1848+$

13. Oxelytrum anticola (Guérin-Méneville, 1855) +

Material examinado. 1 ㅇ, Piura, Ayabaca, Bosque de neblina de Cuyas, estrato bajo, $5^{\circ} 10^{\prime} 51^{\prime \prime} \mathrm{S}-80^{\circ} 37^{\prime} 31^{\prime \prime} \mathrm{O}, 2,122 \mathrm{msnm}, 23-$ II-2019, colecta manual, G. Juárez.

COMENTARIO. Especie cuya distribución abarca Ecuador, Boliviay Perú (Bustamante-Navarrete et al., 2017). Con los datos presentados aquí la especie amplía su distribución dentro de región Piura, ya que solo se citaba para la provincia de Huancabamba (JuárezNoé \& González-Coronado, 2019). El espécimen examinado se colectó sobre cadáver de Sus scrofa domestica Linnaeus, 1758.

Familia Staphylinidae Latreille, 1802

Subfamilia Staphylininae Latreille, 1802

Género Belonuchus Bordmann, 1837

14. Belonuchus sin identificar sp. 1.

Material examinado. $1 \hat{\jmath}$, Piura, Ayabaca, Bosque de neblina de Cuyas, estrato bajo, $5^{\circ} 10^{\prime} 51^{\prime \prime} \mathrm{S}-80^{\circ} 37^{\prime} 31^{\prime \prime} \mathrm{O}, 2,176 \mathrm{msnm}, 11$ III-2018, colecta manual, G. Juárez.

Comentario. El género Belonuchus se distribuye en todas las regiones zoogeográficas del mundo, principalmente en la región neotropical y oriental (Chani-Posse, 2011). Nuevo registro del género para la región Piura (Juárez-Noé \& González-Coronado, 2019). Los individuos examinados se colectaron a ras de suelo confundidos entre la hojarasca.

Género Platydracus Thomson, 1858

15. Platydracus sin identificar sp. 1.

Material examinado. $1 \hat{\jmath}$, Piura, Ayabaca, Bosque de neblina de Cuyas, estrato bajo, $5^{\circ} 10^{\prime} 51^{\prime \prime} \mathrm{S}-80^{\circ} 37^{\prime} 31^{\prime \prime} \mathrm{O}, 2,183 \mathrm{msnm}, 14-$ VI-2016, colecta manual, G. Juárez.

COMENTARIo. Nuevo registro del género para la región Piura (JuárezNoé \& González-Coronado, 2019). Los individuos examinados se colectaron a ras de suelo confundidos entre la hojarasca.

Familia Passalidae Leach, 1815

Género Passalus Fabricius, 1792

16. Passalus sin identificar sp. 1.

Material examinado. 1 , Piura, Ayabaca, Bosque de neblina de Cuyas, estrato medio, $05^{\circ} 10^{\prime} 51^{\prime \prime} \mathrm{S}-80^{\circ} 37^{\prime} 31^{\prime \prime} \mathrm{O}, 2,199 \mathrm{msnm}, 05$ XI-2019, colecta manual, G. Juárez; 1 \&, Piura, Ayabaca, Bosque de neblina de Cuyas, estrato medio, $5^{\circ} 10^{\prime} 51^{\prime \prime} \mathrm{S}-80^{\circ} 37^{\prime} 31^{\prime \prime} \mathrm{O}$, 2,431 msnm, 12-VII-2018, colecta manual, G. Juárez.

Comentario. El individuo examinado se colecto en el interior de tronco en descomposición.

Familia Lucanidae Latreille, 1804

Subfamilia Lucaninae Latreille, 1804

Género Sphaenognathus Buquet, 1838

17. Sphaenognathus (Chiasognathinus) xerophilus Bartolozzi \& Onore, 2006 *

Material examinado. 1 $\hat{\text { a }}$, Piura, Ayabaca, Bosque de neblina de Cuyas, estrato alto, $05^{\circ} 10^{\prime} 51^{\prime \prime} \mathrm{S}-80^{\circ} 37^{\prime} 31^{\prime \prime} \mathrm{O}, 2,733 \mathrm{msnm}, 25$ VI-2016, colecta manual, G. Juárez, 005 LUC-GJN.
Comentario. Especie citada como Sphaenognathus sp en Juárez \& González (2016a). Todas las especies del género Sphaenognathus se distribuyen en las regiones de altas montañas de los andes en Sudamérica (Bolivia, Ecuador, Colombia, Venezuela y Perú), entre los 2,000 msnm hasta los casi 3,500 msnm (Bartolozzi \& Onore, 2006). En la región Piura se distribuye en los bosques montanos de las provincias de Huancabamba y Ayabaca (JuárezNoé \& González-Coronado, 2019).

Subfamilia Syndesinae MacLeay, 1819

Género Psilodon Perty, 1830

18. Psilodon sin identificar sp. 1.

Material examinado. $1 \hat{\jmath}$, Piura, Ayabaca, Bosque de neblina de Cuyas, estrato medio, $05^{\circ} 10^{\prime} 51^{\prime \prime} \mathrm{S}-80^{\circ} 37^{\prime} 31^{\prime \prime} \mathrm{O}, 2,563 \mathrm{msnm}$, 25-VI-2016, colecta manual, G. Juárez.

ComentARIo. El género Psilodon es netamente neotropical habitando zonas de alta montaña de Sudamérica y esta citada para Brasil, Ecuador, Colombia, Bolivia, Argentina y Perú (Paulsen, 2010; Juárez, 2014). Para la región Piura se cita para la provincia de Ayabaca (Juárez-Noé \& González-Coronado, 2019). El espécimen fue colectado en el interior de tronco en descomposición de Myrsine oligophylla Zahlbruckner (Prumilaceae) (Juárez, 2014).

Familia Scarabaeidae Latreille, 1802

Subfamilia Scarabaeinae Latreille, 1802

Género Onthophagus Latreille, 1802

19. Onthophagus $\sin$ identificar sp. 1.

Material examinado. 1 $\hat{\text { }}$, Piura, Ayabaca, Bosque de neblina de Cuyas, estrato medio, $05^{\circ} 10^{\prime} 51^{\prime \prime} \mathrm{S}-80^{\circ} 37^{\prime} 31^{\prime \prime} \mathrm{O}, 2,563 \mathrm{msnm}$, 25-VI-2016, trampa pitfall, G. Juárez.

Comentario. Para la región Piura se cita a Onthophagus confusus Boucomont, 1932 reportado desde la provincia de Morropón en bosques estacionalmente secos de colina (Juárez-Noé \& González-Coronado, 2019).

Género Cryptocanthon Balthasar, $1942+$

20. Cryptocanthon paradoxus Balthasar, $1942+$

Material examinado. 1 \% , Piura, Ayabaca, Bosque de neblina de Cuyas, estrato medio, $5^{\circ} 10^{\prime} 51^{\prime \prime} \mathrm{S}-80^{\circ} 37^{\prime} 31^{\prime \prime} \mathrm{O}, 2,312 \mathrm{msnm}$, 09-III-2018, trampa pitfall, G. Juárez.

Comentario. Las especies de Cryptocanthon tienen amplia distribución en los trópicos del nuevo mundo habitando bosques lluviosos, bosques montanos y altoandinos desde Brasil hasta México (Cook, 2002). En la región Piura $C$. paradoxus se registra para los bosques montanos de la provincia de Ayabaca (Juárez-Noé \& González-Coronado, 2019).

Género Uroxys Westwood, 1842

21. Uroxys sin identificar sp. 1.

Material examinado. $1 \hat{\jmath}$, Piura, Ayabaca, Bosque de neblina de Cuyas, estrato medio, $5^{\circ} 10^{\prime} 51^{\prime \prime} \mathrm{S}-80^{\circ} 37^{\prime} 31^{\prime \prime} \mathrm{O}, 2,303 \mathrm{msnm}$, 29-V-2016, trampa pitfall, G. Juárez.

Comentario. Las especies del género Uroxys se distribuyen en áreas tropicales y subtropicales húmedas de América desde Argentina hasta México exceptuando las Antillas Mayores (Solís \& Kohlmann, 2013).

Género Dichotomius Hope, $1838+$

22. Dichotomius cotopaxi (Guerin, 1855) +

Material examinado. 1 ㅇ, Piura, Ayabaca, Bosque de neblina de Cuyas, estrato bajo, $05^{\circ} 10^{\prime} 51^{\prime \prime} \mathrm{S}-80^{\circ} 37^{\prime} 31^{\prime \prime} \mathrm{O}, 2,012 \mathrm{msnm}, 12$ VI-2018, trampa pitfall, G. Juárez; 1 +, Piura, Ayabaca, Bosque de neblina de Cuyas, estrato medio, $5^{\circ} 10^{\prime} 51^{\prime \prime} \mathrm{S}-80^{\circ} 37^{\prime} 31^{\prime \prime} \mathrm{O}$, 2,425 msnm, 18-X-2018, trampa pitfall, G. Juárez; 1 ô, 1 우, Piura, Ayabaca, Bosque de neblina de Cuyas, estrato medio, $05^{\circ} 10^{\prime} 51^{\prime \prime} \mathrm{S}-80^{\circ} 37^{\prime} 31^{\prime \prime} \mathrm{O}, 2,311 \mathrm{msnm}, 21-\mathrm{IV}-2019$, trampa pitfall, G. Juárez. 
Comentario. La especie se distribuye en Ecuador y Perú entre los 2,200 msnm hasta los 3,100 msnm (Arias-Buriticá, 2011). Para la región Piura se distribuye en bosques montanos de la provincia de Ayabaca (Juárez-Noé \& González-Coronado, 2019).

Género Canthon Hoffmannsegg, 1817

23. Canthon fuscipes Erichson, 1847

Material examinado. 2 우, $2 \hat{\jmath} \hat{\delta}$, Piura, Ayabaca, Bosque de neblina de Cuyas, estrato bajo, $05^{\circ} 10^{\prime} 51^{\prime \prime} \mathrm{S}-80^{\circ} 37^{\prime} 31^{\prime \prime} \mathrm{O}, 2,021$ msnm, 22-III-2018, trampa pitfall, G. Juárez; 1 \&, Piura, Ayabaca, Bosque de neblina de Cuyas, estrato bajo, $5^{\circ} 10^{\prime} 51^{\prime \prime} \mathrm{S}-80^{\circ} 37^{\prime} 31^{\prime \prime} \mathrm{O}$, 2,101 msnm, 29-IV-2019, trampa pitfall, U. González; 1 ð, 1 +, Piura, Ayabaca, Bosque de neblina de Cuyas, estrato alto, $05^{\circ} 10^{\prime} 51^{\prime \prime} \mathrm{S}-80^{\circ} 37^{\prime} 31^{\prime \prime} \mathrm{O}, 2,603 \mathrm{msnm}, 14-\mathrm{VI}-2019$, trampa pitfall, G. Juárez; 1 q, Piura, Ayabaca, Bosque de neblina de Cuyas, estrato alto, $5^{\circ} 10^{\prime} 51^{\prime \prime} \mathrm{S}-80^{\circ} 37^{\prime} 31^{\prime \prime} \mathrm{O}, 2,612 \mathrm{msnm}, 3-\mathrm{IX}-2019$, trampa pitfall, G. Juárez.

Comentario. Especie de amplia distribución en la región Piura abarcando todas las provincias y paisajes ecológicos (JuárezNoé \& González-Coronado, 2019).

Género Onoreidium Vaz de Mello, 2008

24. Onoreidium cristatum (Arrow, 1931)

Material examinado. 1 d, Piura, Ayabaca, Bosque de neblina de Cuyas, estrato medio, $05^{\circ} 10^{\prime} 51^{\prime \prime} \mathrm{S}-80^{\circ} 37^{\prime} 31^{\prime \prime} \mathrm{O}, 2,401 \mathrm{msnm}, 22-$ VII-2018, trampa pitfall, U. González; 1 \&,Piura,Ayabaca, Bosque de neblina de Cuyas, estrato medio, $5^{\circ} 10^{\prime} 51^{\prime \prime} \mathrm{S}-80^{\circ} 37^{\prime} 31^{\prime \prime} \mathrm{O}$, 2,412 msnm, 13-XII-2019, trampa pitfall, G. Juárez.

Comentario. Especie citada también para la región Piura en bosques húmedos de montaña de la provincia de Huancabamba (Juárez-Noé \& González-Coronado, 2019).

Género Ontherus Erichson, $1847+$

25. Ontherus (Caelontherus) howdeni Génier, $1996+$

Material examinado. 1 ô, Piura, Ayabaca, Bosque de neblina de Cuyas, estrato medio, $05^{\circ} 10^{\prime} 51^{\prime \prime} \mathrm{S}-80^{\circ} 37^{\prime} 31^{\prime \prime} \mathrm{O}, 2,521 \mathrm{msnm}$, 22-VII-2018, trampa pitfall, G. Juárez.

Comentario. La especie se distribuye en Ecuador y Perú (regiones de Piura y Junín). Para la región Piura se registra en bosques montanos de las provincias de Ayabaca y Huancabamba (Génier, 1996; Juárez-Noé \& González-Coronado, 2019).

26. Ontherus (Planontherus) rectus Génier, $1996+*$

Material examinado. $1 \hat{\sigma}$, Piura, Ayabaca, Bosque de neblina de Cuyas, estrato medio, $05^{\circ} 10^{\prime} 51^{\prime \prime} \mathrm{S}-80^{\circ} 37^{\prime} 31^{\prime \prime} \mathrm{O}, 2,521 \mathrm{msnm}$, 22-VII-2018, trampa pitfall, G. Juárez.

Comentario. Especie conocida únicamente de Perú y se distribuye en bosques montanos de las provincias de Ayabaca y Huancabamba de la región Piura (Génier, 1996; Juárez-Noé \& González-Coronado, 2019).

Género Scatimus Erichson, 1847

27. Scatimus monstrosus Balthasar, 1939 (Fig. 3)

Material examinado. $1 \hat{\delta}$, Piura, Ayabaca, Bosque de neblina de Cuyas, estrato medio, $05^{\circ} 10^{\prime} 51^{\prime \prime} \mathrm{S}-80^{\circ} 37^{\prime} 31^{\prime \prime} \mathrm{O}, 2,521 \mathrm{msnm}$, 11-VII-2016, trampa pitfall, G. Juárez.

Comentario. Especie que se distribuye en Ecuador y Perú (regiones de Piura y Lima) (Génier \& Kohlmann, 2003; Juárez-Noé \& González-Coronado, 2019). En la región Piura se distribuye en bosques montanos de la provincia de Ayabaca (Juárez-Noé \& González-Coronado, 2019).

Subfamilia Melolonthinae Leach, 1819

Género Plectris Le Peletier \& Audinet-Serville, 1828

28. Plectris sin identificar sp. 1.

Material examinado. 1 , Piura, Ayabaca, Bosque de neblina de Cuyas, estrato medio, $05^{\circ} 10^{\prime} 51^{\prime \prime} \mathrm{S}-80^{\circ} 37^{\prime} 31^{\prime \prime} \mathrm{O}, 2,403 \mathrm{msnm}, 12-$
IV-2019, colecta manual, G. Juárez; 1 đ̊, Piura, Ayabaca, Bosque de neblina de Cuyas, estrato medio, $05^{\circ} 10^{\prime} 51^{\prime \prime} \mathrm{S}-80^{\circ} 37^{\prime} 31^{\prime \prime} \mathrm{O}$, 2,431 msnm, 09-VI-2019, colecta manual, G. Juárez.

Comentario. Hasta el momento para la región Piura el género Plectris se cita para la provincia de Ayabaca en bosques de neblina (Juárez-Noé \& González-Coronado, 2019).

\section{Género Phyllophaga Harris, 1827}

29. Phyllophaga sin identificar sp. 1.

Material eXaminado. 1 $\hat{\text { }}$, Piura, Ayabaca, Bosque de neblina de Cuyas, estrato medio, $05^{\circ} 10^{\prime} 51^{\prime \prime} \mathrm{S}-80^{\circ} 37^{\prime} 31^{\prime \prime} \mathrm{O}, 2,309 \mathrm{msnm}, 12-$ IV-2019, colecta manual, G. Juárez; 1 đ̃, Piura, Ayabaca, Bosque de neblina de Cuyas, estrato medio, $05^{\circ} 10^{\prime} 51^{\prime \prime} \mathrm{S}-80^{\circ} 37^{\prime} 31^{\prime \prime} \mathrm{O}$, 2,345 msnm, 09-VI-2019, colecta manual, G. Juárez.

Comentario. Hasta el momento para la región Piura el género Phyllophaga se cita para la provincia de Ayabaca en bosques de neblina (Juárez-Noé \& González-Coronado, 2019).

Subfamilia Rutelinae MacLeay, 1819

Género Platycoelia Dejean, 1833

30. Platycoelia marginata Burmeister, 1844

Material eXaminado. $1 \hat{\delta}$, Piura, Ayabaca, Bosque de neblina de Cuyas, estrato medio, $05^{\circ} 10^{\prime} 51^{\prime \prime} \mathrm{S}-80^{\circ} 37^{\prime} 31^{\prime \prime} \mathrm{O}, 2,430 \mathrm{msnm}$, 14-IV-2016, colecta manual, G. Juárez, 023 SCA-GJN.

Comentario. Especie citada como Platycoelia sp en Juárez \& González (2016a). Platycoelia marginata se distribuye en las laderas orientales de los Andes desde el oeste de Venezuela hasta el norte de Perú (Smith, 2003). Para la región Piura se registra en bosques secos interandinos y bosques montanos de las provincias de Ayabaca y Huancabamba (Juárez-Noé \& González-Coronado, 2019).

Género Mecopelidnota F. Bates, 1904.

31. Mecopelidnota marxi Soula, 2008 (Fig. 4)

Material eXaminado. 1 ô, Piura, Ayabaca, Bosque de neblina de Cuyas, estrato medio, $05^{\circ} 10^{\prime} 51^{\prime \prime} \mathrm{S}-80^{\circ} 37^{\prime} 31^{\prime \prime} \mathrm{O}, 2,430 \mathrm{msnm}$, 14-IV-2016, colecta manual, U. González, 027 SCA-GJN; $1 \hat{\partial}$, Piura, Ayabaca, Bosque de neblina de Cuyas, estrato medio, $05^{\circ} 10^{\prime} 51^{\prime \prime} \mathrm{S}-80^{\circ} 37^{\prime} 31^{\prime \prime} \mathrm{O}, 2,511 \mathrm{msnm}, 14-\mathrm{XI}-2018$, trampa luz, G. Juárez; 1 సै, Piura, Ayabaca, Bosque de neblina de Cuyas, estrato medio, $05^{\circ} 10^{\prime} 51^{\prime \prime} \mathrm{S}-80^{\circ} 37^{\prime} 31^{\prime \prime} \mathrm{O}, 2,430 \mathrm{msnm}$, 03-II-2019, colecta manual, G. Juárez.

Comentario. Con la consulta de Soula (2008), se llegó a la conclusión que el género Strigidia Dejean, 1833 citado en Juárez \& González (2016a) se trata de M. marxi. Esta especie en la región Piura habita bosques estacionalmente secos, bosques secos interandinos y bosque húmedos de montaña en las provincias de Piura, Sullana y Huancabamba (Juárez-Noé \& González-Coronado, 2018, 2019). Con los datos presentados aquí amplía su distribución a la provincia de Ayabaca.

Género Thyriochlorata Ohaus, 1915

32. Thyriochlorata villosa (Ohaus, 1908) *

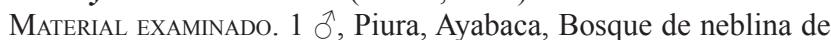
Cuyas, estrato medio, $05^{\circ} 10^{\prime} 51^{\prime \prime} \mathrm{S}-80^{\circ} 37^{\prime} 31^{\prime \prime} \mathrm{O}, 2,445 \mathrm{msnm}$, 14-IV-2016, colecta manual, G. Juárez, 029 SCA-GJN.

Comentario. Con la consulta de Soula (2002), se llegó a la conclusión que el género Pseudothyridium Soula, 2002 citado en Juárez \& González (2016a) se trata de T. villosa. Especie conocida solamente de Perú desde la región Piura (provincia de Huancabamba) (Soula, 2002; Juárez-Noé \& González-Coronado, 2019). Con los datos presentados aquí amplía su distribución a la provincia de Ayabaca.

Género Leucothyreus MacLeay, 1819

33. Leucothyreus sin identificar sp. 1.

Material EXAMinado. $2 \delta^{\lambda} \sigma^{\lambda}$, Piura, Ayabaca, Bosque de neblina de Cuyas, estrato bajo, $05^{\circ} 10^{\prime} 51^{\prime \prime} \mathrm{S}-80^{\circ} 37^{\prime} 31^{\prime \prime} \mathrm{O}, 2,000 \mathrm{msnm}, 21$ - 
X-2018, colecta manual, G. Juárez; 1 đે, Piura, Ayabaca, Bosque de neblina de Cuyas, estrato bajo, 05 $10^{\prime} 51^{\prime \prime} \mathrm{S}-80^{\circ} 37^{\prime} 31^{\prime \prime} \mathrm{O}$, 2,098 msnm, 22-X-2018, trampa luz, U. González; 1 , Piura, Ayabaca, Bosque de neblina de Cuyas, estrato medio, $05^{\circ} 10^{\prime} 51^{\prime \prime} \mathrm{S}-80^{\circ} 37^{\prime} 31^{\prime \prime} \mathrm{O}, 2,338 \mathrm{msnm}, 11-\mathrm{II}-2019$, colecta manual, G. Juárez; 1 \&, Piura, Ayabaca, Bosque de neblina de Cuyas, estrato alto, $05^{\circ} 10^{\prime} 51^{\prime \prime} \mathrm{S}-80^{\circ} 37^{\prime} 31^{\prime \prime} \mathrm{O}, 2,611 \mathrm{msnm}, 28$ VII-2019, colecta manual, G. Juárez.

COMENTARIo. Para la región Piura también se han colectado individuos diferentes del género Leucothyreus en bosques estacionalmente secos y desierto costero mencionados como Leucothyreus sp. 2 por Juárez-Noé \& González-Coronado (2019).

Género Paranomala Casey, $1915+$

34. Paranomala undulata peruviana Guérin-Méneville, $1831+$ (Fig. 5)

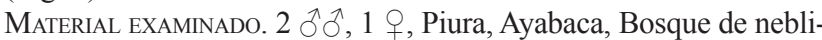
na de Cuyas, estrato bajo, $05^{\circ} 10^{\prime} 51^{\prime \prime} \mathrm{S}-80^{\circ} 37^{\prime} 31^{\prime \prime} \mathrm{O}, 2,000 \mathrm{msnm}$, 21-X-2018, trampa luz, U. González; 1 §̃, Piura, Ayabaca, Bosque de neblina de Cuyas, estrato bajo, $05^{\circ} 10^{\prime} 51^{\prime \prime} \mathrm{S}-80^{\circ} 37^{\prime} 31^{\prime \prime} \mathrm{O}$, 2,098 msnm, 22-X-2018, trampa luz, U. González; 1 ㅇ, 1 స, Piura, Ayabaca, Bosque de neblina de Cuyas, estrato medio, $05^{\circ} 10^{\prime} 51^{\prime \prime} \mathrm{S}-80^{\circ} 37^{\prime} 31^{\prime \prime} \mathrm{O}, 2,411 \mathrm{msnm}, 03-\mathrm{XII}-2019$, colecta manual, U. González; 1 ㅇ, Piura, Ayabaca, Bosque de neblina de Cuyas, estrato alto, $05^{\circ} 10^{\prime} 51^{\prime \prime} \mathrm{S}-80^{\circ} 37^{\prime} 31^{\prime \prime} \mathrm{O}, 2,611 \mathrm{msnm}, 28$ VII-2019, colecta manual, G. Juárez; 2 ふै ô, Piura, Ayabaca, Bosque de neblina de Cuyas, estrato alto, $05^{\circ} 10^{\prime} 51^{\prime \prime} \mathrm{S}-80^{\circ} 37^{\prime} 31^{\prime \prime} \mathrm{O}$, 2,711 msnm, 21-X-2018, colecta manual, G. Juárez.

Comentario. Especie que se distribuye en Ecuador, Chile y Perú (Mondaca, 2012; Ratcliffe et al., 2015). Es de amplia distribución en la región Piura abarcando todas las provincias y paisajes ecológicos (Juárez-Noé \& González-Coronado, 2018, 2019).

Género Macraspis MacLeay, $1819+$

35. Macraspis festiva Burmeister, $1844+$

Material examinado. 1 , Piura, Ayabaca, Bosque de neblina de Cuyas, estrato medio, $05^{\circ} 10^{\prime} 51^{\prime \prime} \mathrm{S}-80^{\circ} 37^{\prime} 31^{\prime \prime} \mathrm{O}, 2,345 \mathrm{msnm}$, 21-X-2018, colecta manual, G. Juárez.

Comentario. Especie que se distribuye en bosques montanos y lluviosos de Venezuela, Colombia, Ecuador, Bolivia, Brasil, Paraguay y Perú (Soula, 1999).

Subfamilia Dynastinae MacLeay, 1819

Género Ancognatha Erichson, 1847

36. Ancognatha scarabaeoides Erichson, 1847 (Fig. 6)

Material examinado. 1 $\hat{\delta}$, Piura, Ayabaca, Bosque de neblina de Cuyas, estrato medio, $05^{\circ} 10^{\prime} 51^{\prime \prime} \mathrm{S}-80^{\circ} 37^{\prime} 31^{\prime \prime} \mathrm{O}, 2,665 \mathrm{msnm}$, 21-X-2018, colecta manual, G. Juárez.

Comentario. En Perú esta especie tiene amplia distribución en zonas altoandinas desde los 2,000 msnm hasta casi los 4,000 msnm (Figueroa \& Ratcliffe, 2016). En la región Piura se distribuye en bosques secos interandinos y bosques montanos de las provincias de Ayabaca y Huancabamba (Juárez-Noé \& González-Coronado, 2019).

37. Ancognatha vulgaris Arrow, 1911 (Fig. 7)

Material examinado. $1 \hat{\jmath}$, Piura, Ayabaca, Bosque de neblina de Cuyas, estrato bajo, $05^{\circ} 10^{\prime} 51^{\prime \prime} \mathrm{S}-80^{\circ} 37^{\prime} 31^{\prime \prime} \mathrm{O}, 2,100 \mathrm{msnm}, 26-$ I-2018, colecta manual, G. Juárez; 1 ก๊, Piura, Ayabaca, Bosque de neblina de Cuyas, estrato medio, $05^{\circ} 10^{\prime} 51^{\prime \prime} \mathrm{S}-80^{\circ} 37^{\prime} 31^{\prime \prime} \mathrm{O}$, 2,665 msnm, 30-VIII-2019, colecta manual, U. González.
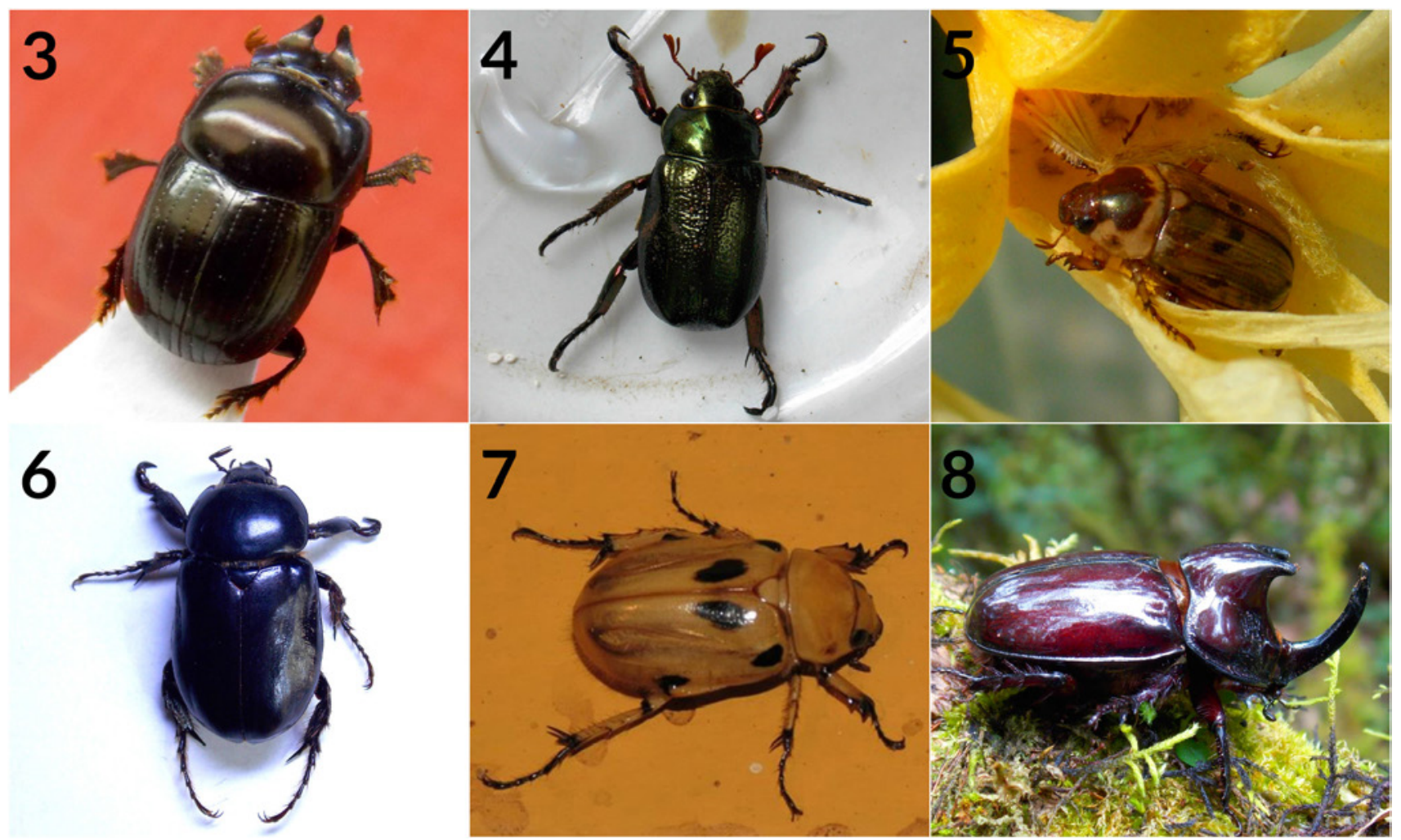

Figs. 3-8.- Habitus. 3. Scatimus monstrosus Balthasar, 1939. 4. Mecopelidnota marxi Soula, 2008. 5. Paranomala undulata peruviana Guérin-Méneville, 1831. 6. Ancognatha scarabaeoides Erichson, 1847. 7. Ancognatha vulgaris Arrow, 1911.8. Megaceras morpheus Burmeister, 1847. Fotografías 3-6, 8 por G. Juárez y U. González; fotografía 7 por F. Angulo].

Figs. 3-8.- Habitus. 3. Scatimus monstrosus Balthasar, 1939. 4. Mecopelidnota marxi Soula, 2008. 5. Paranomala undulata peruviana Guérin-Méneville, 1831. 6. Ancognatha scarabaeoides Erichson, 1847. 7. Ancognatha vulgaris Arrow, 1911.8. Megaceras morpheus Burmeister, 1847. Photographs 3-6, 8 by G. Juárez and U. González; photography 7 by F. Angulo]. 
Comentario. En Perú esta especie tiene distribución tanto en zonas de bosques estacionalmente secos de montaña y en zonas altoandinas desde los $500 \mathrm{msnm}$ hasta casi 3,000 msnm (Figueroa \& Ratcliffe, 2016). En la región Piura se distribuye en bosques secos interandinos y bosques montanos de las provincias de Ayabaca y Huancabamba (Juárez-Noé \& González-Coronado, 2019).

Género Golofa Hope, 1837

38. Golofa aegeon (Drury, 1773) +

Material examinado. $1 \hat{\partial}$, Piura, Ayabaca, Bosque de neblina de Cuyas, estrato bajo, $05^{\circ} 10^{\prime} 51^{\prime \prime} \mathrm{S}-80^{\circ} 37^{\prime} 31^{\prime \prime} \mathrm{O}, 2,197 \mathrm{msnm}, 25$ -

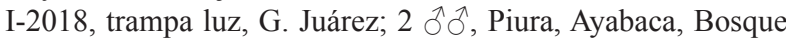
de neblina de Cuyas, estrato medio, $05^{\circ} 10^{\prime} 51^{\prime \prime} \mathrm{S}-80^{\circ} 37^{\prime} 31^{\prime \prime} \mathrm{O}$, 2,595 msnm, 29-VIII-2019, colecta manual, G. Juárez; 1 đ, Piura, Ayabaca, Bosque de neblina de Cuyas, estrato bajo, $05^{\circ} 10^{\prime} 51^{\prime \prime} \mathrm{S}-80^{\circ} 37^{\prime} 31^{\prime \prime} \mathrm{O}, 2,100 \mathrm{msnm}, 12-\mathrm{XII}-2019$, colecta manual, U. González; 1 సै, Piura, Ayabaca, Bosque de neblina de Cuyas, estrato medio, $05^{\circ} 10^{\prime} 51^{\prime \prime} \mathrm{S}-80^{\circ} 37^{\prime} 31^{\prime \prime} \mathrm{O}, 2,665$ msnm, 15-IV-2019, colecta manual, G. Juárez.

Comentario. Especie que se distribuye en Ecuador, Colombia y Perú (Lachaume, 1985). Para la región Piura se cita para la provincia de Huancabamba habitando bosques húmedos de montaña (JuárezNoé \& González-Coronado, 2019). Con los datos presentados aquí amplía su distribución a la provincia de Ayabaca.

39. Golofa eacus Burmeister, 1847

Material examinado. $1 \hat{\jmath}$, Piura, Ayabaca, Bosque de neblina de Cuyas, estrato bajo, $05^{\circ} 10^{\prime} 51^{\prime \prime} \mathrm{S}-80^{\circ} 37^{\prime} 31^{\prime \prime} \mathrm{O}, 2,197 \mathrm{msnm}, 25-\mathrm{I}-$ 2018, trampa luz, G. Juárez; 1 đ̊, Piura, Ayabaca, Bosque de neblina de Cuyas, estrato medio, $05^{\circ} 10^{\prime} 51^{\prime \prime} \mathrm{S}-80^{\circ} 37^{\prime} 31^{\prime \prime} \mathrm{O}, 2,595 \mathrm{msnm}$, 29-VIII-2019, trampa luz, U. González; 1 ô, Piura, Ayabaca, Bosque de neblina de Cuyas, estrato alto, $05^{\circ} 10^{\prime} 51^{\prime \prime} \mathrm{S}-80^{\circ} 37^{\prime} 31^{\prime \prime} \mathrm{O}$, 2,703 msnm, 12-XII-2019, colecta manual, G. Juárez.

Comentario. Especie que se distribuye en Ecuador, Colombia, Venezuela, Bolivia y Perú (Lachaume, 1985). Para la región Piura se cita para las provincias de Huancabamba y Ayabaca habitando bosques húmedos de montaña (Juárez-Noé \& González-Coronado, 2019).

40. Golofa limogesi Ratcliffe \& Le Tirant, $2017+*$

Material examinado. $1 \hat{\delta}$, Piura, Ayabaca, Bosque de neblina de Cuyas, estrato medio, $05^{\circ} 10^{\prime} 51^{\prime \prime} \mathrm{S}-80^{\circ} 37^{\prime} 31^{\prime \prime} \mathrm{O}, 2,550 \mathrm{msnm}$, 10-IX-2019, trampa luz, G. Juárez; 1 ڤึ, Piura, Ayabaca, Bosque de neblina de Cuyas, estrato alto, $05^{\circ} 10^{\prime} 51^{\prime \prime} \mathrm{S}-80^{\circ} 37^{\prime} 31^{\prime \prime} \mathrm{O}$, 2,875 msnm, 10-IX-2019, colecta manual, G. Juárez.

Comentario. Especie conocida solamente en Perú desde la región Piura (provincias de Ayabaca y Huancabamba) habitando bosques montanos (Ratcliffe \& Le Tirant, 2017; Juárez-Noé \& González-Coronado, 2019).

Género Megaceras Hope, 1837

41. Megaceras morpheus Burmeister, 1847 (Fig. 8)

Material examinado. $2 \hat{\partial} \hat{\partial}$, Piura, Ayabaca, Bosque de neblina de Cuyas, estrato medio, $05^{\circ} 10^{\prime} 51^{\prime \prime} \mathrm{S}-80^{\circ} 37^{\prime} 31^{\prime \prime} \mathrm{O}, 2,589 \mathrm{msnm}, 10$ I-2018, trampa luz, U. González; 1 đ̂, 1 †, Piura, Ayabaca, Bosque de neblina de Cuyas, estrato medio, $05^{\circ} 10^{\prime} 51^{\prime \prime} \mathrm{S}-80^{\circ} 37^{\prime} 31^{\prime \prime} \mathrm{O}$, 2,466 msnm, 09-V-2016, colecta manual, G. Juárez, 045 SCA-

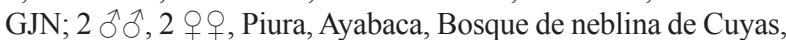
estrato bajo, $05^{\circ} 10^{\prime} 51^{\prime \prime} \mathrm{S}-80^{\circ} 37^{\prime} 31^{\prime \prime} \mathrm{O}, 2,101 \mathrm{msnm}, 23-\mathrm{IV}-2019$, trampa luz, U. González; 1 กิ, 1 †, Piura, Ayabaca, Bosque de neblina de Cuyas, estrato medio, $05^{\circ} 10^{\prime} 51^{\prime \prime} \mathrm{S}-80^{\circ} 37^{\prime} 31^{\prime \prime} \mathrm{O}$,

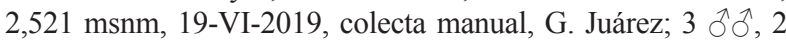
우, Piura, Ayabaca, Bosque de neblina de Cuyas, estrato alto, $05^{\circ} 10^{\prime} 51^{\prime \prime} \mathrm{S}-80^{\circ} 37^{\prime} 31^{\prime \prime} \mathrm{O}, 2,875 \mathrm{msnm}, 2-\mathrm{IV}-2019$, trampa luz, G. Juárez; 1 $\widehat{\jmath}$, Piura, Ayabaca, Bosque de neblina de Cuyas, estrato alto, $05^{\circ} 10^{\prime} 51^{\prime \prime} \mathrm{S}-80^{\circ} 37^{\prime} 31^{\prime \prime} \mathrm{O}, 2,900 \mathrm{msnm}, 14-\mathrm{XII}-2019$, colecta manual, G. Juárez.

COMENTARIO. Especie que se distribuye en zonas andinas y zonas de altas montañas de Venezuela, Ecuador, Brasil, Argentina, Colombia, Panamá y Honduras (Sanabria-García, 2012). Para la región Piura se cita en bosques montanos de las provincias de Ayabaca y Huancabamba (Juárez-Noé \& González-Coronado, 2019).

Género Heterogomphus Burmeister, 1847

42. Heterogomphus rubripennis Prell, 1912 (Fig. 9)

Material examinado. $1 \hat{\sigma}$, Piura, Ayabaca, Bosque de neblina de Cuyas, estrato medio, $05^{\circ} 10^{\prime} 51^{\prime \prime} \mathrm{S}-80^{\circ} 37^{\prime} 31^{\prime \prime} \mathrm{O}, 2,593 \mathrm{msnm}$, 15-V-2016, colecta manual, G. Juárez, 049 SCA-GJN; 1 \%, Piura, Ayabaca, Bosque de neblina de Cuyas, estrato alto, $05^{\circ} 10^{\prime} 51^{\prime \prime} \mathrm{S}-80^{\circ} 37^{\prime} 31^{\prime \prime} \mathrm{O}, 2,690 \mathrm{msnm}, 25-\mathrm{VIII}-2019$, colecta manual, G. Juárez.

Comentario. La especie en la región Piura se distribuye en los bosques montanos de la provincia de Ayabaca (Juárez-Noé \& González-Coronado, 2019).

Género Coelosis Hope, 1837

43. Coelosis biloba (Linnaeus, 1767)

Material examinado. 1 $\widehat{\pi}$, Piura, Ayabaca, Bosque de neblina de Cuyas, estrato medio, $05^{\circ} 10^{\prime} 51^{\prime \prime} \mathrm{S}-80^{\circ} 37^{\prime} 31^{\prime \prime} \mathrm{O}, 2,420$ msnm, 29-VII-2018, colecta manual, G. Juárez; 1 đ, Piura, Ayabaca, Bosque de neblina de Cuyas, estrato alto, $05^{\circ} 10^{\prime} 51^{\prime \prime} \mathrm{S}-80^{\circ} 37^{\prime} 31^{\prime \prime} \mathrm{O}, 2,734 \mathrm{msnm}, 03-\mathrm{XII}-2019$, colecta manual, G. Juárez.

Comentario. Esta especie se distribuye desde México hasta el sur de Argentina habitando sabanas, áreas degradadas y bosques de alta montaña (Endrödi, 1985; Ratcliffe et al., 2013). En la región Piura se distribuye en bosques montanos de las provincias de Ayabaca y Huancabamba (Juárez-Noé \& González-Coronado, 2019).

Género Stenocrates Burmeister, 1847

44. Stenocrates sin identificar sp. 1.

Material examinado. $1 \hat{\delta}, 1$ 을 Piura, Ayabaca, Bosque de neblina de Cuyas, estrato medio, $05^{\circ} 10^{\prime} 51^{\prime \prime} \mathrm{S}-80^{\circ} 37^{\prime} 31^{\prime \prime} \mathrm{O}, 2,420 \mathrm{msnm}$, 03-III-2018, trampa luz, G. Juárez; 1 ڤึ, Piura, Ayabaca, Bosque de neblina de Cuyas, estrato alto, $05^{\circ} 10^{\prime} 51^{\prime \prime} \mathrm{S}-80^{\circ} 37^{\prime} 31^{\prime \prime} \mathrm{O}$, 2,677 msnm, 13-IX-2019, colecta manual, G. Juárez.

Comentario. El género Stenocrates se distribuye desde México hasta Argentina con amplía dominancia en Sudamérica, habitando bosques amazónicos, montanos y altoandinos (Ratcliffe, 2015). Nuevo registro del género para la región Piura (Juárez-Noé \& González-Coronado, 2019).

Género Strategus Kirby, $1828+$

45. Strategus aloeus (Linnaeus, 1758) +

Material examinado. $1 \hat{\jmath}$, Piura, Ayabaca, Bosque de neblina de Cuyas, estrato medio, $05^{\circ} 10^{\prime} 51^{\prime \prime} \mathrm{S}-80^{\circ} 37^{\prime} 31^{\prime \prime} \mathrm{O}, 2,578$ msnm, 21-II-2019, colecta manual, G. Juárez; 1 స, Piura, Ayabaca, Bosque de neblina de Cuyas, estrato alto, $05^{\circ} 10^{\prime} 51^{\prime \prime} \mathrm{S}-80^{\circ} 37^{\prime} 31^{\prime \prime} \mathrm{O}, 2,731 \mathrm{msnm}, 10-\mathrm{IV}-2019$, colecta manual, G. Juárez.

Comentario. Especie que se distribuye desde el sur de los Estados Unidos, América Central hasta Bolivia (Ratcliffe, 1976). En la región Piura se distribuye en bosques montanos de las provincias de Morropón, Ayabaca y Huancabamba (JuárezNoé \& González-Coronado, 2019).

\section{Género Dynastes Kirby, $1825+$}

46. Dynastes neptunus (Quensel, 1806) +

Material examinado. 1 $\lambda$, Piura, Ayabaca, Bosque de neblina de Cuyas, estrato alto, $05^{\circ} 10^{\prime} 51^{\prime \prime} \mathrm{S}-80^{\circ} 37^{\prime} 31^{\prime \prime} \mathrm{O}, 2,703 \mathrm{msnm}, 21$ II-2019, colecta manual, G. Juárez. 
COMENTARIo. Con los datos presentados aquí amplía su distribución a la provincia de Ayabaca, antes citada para la provincia de Huancabamba (Juárez-Noé \& González-Coronado, 2019).

Género Megasoma Kirby, $1825+$

47. Megasoma actaeon (Linnaeus, 1758) +

Material examinado. $1 \hat{\delta}$, Piura, Ayabaca, Bosque de neblina de Cuyas, estrato alto, $05^{\circ} 10^{\prime} 51^{\prime \prime} \mathrm{S}-80^{\circ} 37^{\prime} 31^{\prime \prime} \mathrm{O}, 2,712 \mathrm{msnm}, 21$ II-2019, colecta manual, G. Juárez.

Comentario. Con los datos presentados aquí amplía su distribución a la provincia de Ayabaca, antes citada para la provincia de Morropón (Juárez-Noé \& González-Coronado, 2019).

Subfamilia Cetoniinae Leach, 1815

Género Hoplopyga Thomson, 1880

48. Hoplopyga liturata (Olivier, 1789)

Material examinado. 1 $\widehat{\partial}$, Piura, Ayabaca, Bosque de neblina de Cuyas, estrato bajo, $05^{\circ} 10^{\prime} 51^{\prime \prime} \mathrm{S}-80^{\circ} 37^{\prime} 31^{\prime \prime} \mathrm{O}, 2,004 \mathrm{msnm}, 21-$ II-2016, colecta manual, U. González, 053 SCA-GJN; 1 సे, Piura, Ayabaca, Bosque de neblina de Cuyas, estrato medio, $05^{\circ} 10^{\prime} 51^{\prime \prime} \mathrm{S}-80^{\circ} 37^{\prime} 31^{\prime \prime} \mathrm{O}, 2,302 \mathrm{msnm}, 11-\mathrm{VI}-2019$, colecta manual, U. González.

COMENTARIO. La especie tiene amplia distribución en el neotrópico abarcando desde el sur de México hasta Argentina (Shaughney $\&$ Ratcliffe, 2015). Para la región Piura se cita para la provincia de Huancabamba (Juárez-Noé \& González-Coronado, 2019), con los datos presentados aquí amplía su distribución a la provincia de Ayabaca.

Subfamilia Aphodiinae Leach, 1815

Género Aphodius Illiger, $1798+$

49. Aphodius (Labarrus) pseudolividus Balthasar, $1941+$

Material eXAminado. $2 \hat{\delta} \hat{\jmath}$, Piura, Ayabaca, Bosque de neblina de Cuyas, estrato bajo, $05^{\circ} 10^{\prime} 51^{\prime \prime} \mathrm{S}-80^{\circ} 37^{\prime} 31^{\prime \prime} \mathrm{O}, 2,011 \mathrm{msnm}, 11-\mathrm{III}-$ 2018, trampa luz, U. González; 1 ô, 1 क , Piura, Ayabaca, Bosque de neblina de Cuyas, estrato bajo, $05^{\circ} 10^{\prime} 51^{\prime \prime} \mathrm{S}-80^{\circ} 37^{\prime} 31^{\prime \prime} \mathrm{O}$, 2,121 msnm, 1-VI-2019, colecta manual, G. Juárez; 1 §ิ, 3 우우울. Ayabaca, Bosque de neblina de Cuyas, estrato medio, $05^{\circ} 10^{\prime} 51^{\prime \prime} \mathrm{S}-80^{\circ} 37^{\prime} 31^{\prime \prime} \mathrm{O}, 2,333 \mathrm{msnm}, 17-\mathrm{IV}-2019$, colecta manual, U. González ; 1 §, 1 \&, Piura, Ayabaca, Bosque de neblina de Cuyas, estrato alto, $05^{\circ} 10^{\prime} 51^{\prime \prime} \mathrm{S}-80^{\circ} 37^{\prime} 31^{\prime \prime} \mathrm{O}$, 2,832 msnm, 23-IX-2019, colecta manual, G. Juárez.

COMENTARIO. Especie de amplia distribución mundial registrándose en Australia, Nueva Zelanda, Oceanía, África, América Central y América del Sur (Smith \& Skelley, 2007). En la región Piura se distribuye en todas las provincias habitando desde bosques estacionalmente secos, desierto, manglares, bosques secos interandinos hasta bosques húmedos de montaña (Juárez-Noé \& González-Coronado, 2018, 2019). Algunos de los individuos estudiados se colectaron en heces frescas de ganado vacuno.

Familia Elateridae Leach, 1815

Subfamilia Semiotinae Jakobson, 1913

Género Semiotus Eschscholtz, 1829

50. Semiotus angustus Wells, 2017

Material examinado. 1 , , Piura, Ayabaca, Bosque de neblina de Cuyas, estrato medio, $05^{\circ} 10^{\prime} 51^{\prime \prime} \mathrm{S}-80^{\circ} 37^{\prime} 31^{\prime \prime} \mathrm{O}, 2,579 \mathrm{msnm}$, 15-VI-2016, colecta manual, G. Juárez, 065 ELA-GJN.

Comentario. Especie citada como Semiotus sp en Juárez \& González (2016a). Especie que se distribuye en zonas de bosques montanos y altoandinas de Bolivia y Perú (Wells, 2007). Para la región Piura en bosques húmedos de montaña de la provincia de Ayabaca (Juárez-Noé \& González-Coronado, 2019).

Familia Lycidae Laporte, 1836

Subfamilia Lycinae Laporte, 1836
Género Calopteron Laporte, 1838

51. Calopteron $\sin$ identificar sp. 1.

Material examinado. 1 , Piura, Ayabaca, Bosque de neblina de Cuyas, estrato medio, $05^{\circ} 10^{\prime} 51^{\prime \prime} \mathrm{S}-80^{\circ} 37^{\prime} 31^{\prime \prime} \mathrm{O}, 2,587 \mathrm{msnm}, 15-$ XI-2018, colecta manual, G. Juárez; 1 + , Piura, Ayabaca, Bosque de neblina de Cuyas, estrato medio, $05^{\circ} 10^{\prime} 51^{\prime \prime} \mathrm{S}-80^{\circ} 37^{\prime} 31^{\prime \prime} \mathrm{O}$, 2532 msnm, 20-II-2019, colecta manual, G. Juárez.

Comentario. Iguales individuos también han sido colectados en las provincias de Huancabamba en bosques húmedos de montaña y bosques secos interandinos (Juárez-Noé \& GonzálezCoronado, 2019).

Familia Lampyridae Rafinesque, 1815

Subfamilia Amydetinae Olivier, 1907

Género Cladodes Solier, 1849

52. Cladodes sin identificar sp. 1. (Fig. 10)

Material examinado. 1 , Piura, Ayabaca, Bosque de neblina de Cuyas, estrato medio, $05^{\circ} 10^{\prime} 51^{\prime \prime} \mathrm{S}-80^{\circ} 37^{\prime} 31^{\prime \prime} \mathrm{O}, 2,587 \mathrm{msnm}$, 16-XI-2018, colecta manual, G. Juárez.

Comentario. Hasta el momento es el único individuo colectado del género Cladodes en la región Piura.

Subfamilia Lampyrinae Rafinesque, 1815

Género Photinus Laporte, 1833

53. Photinus sin identificar sp. 1.

Material examinado. 1 , Piura, Ayabaca, Bosque de neblina de Cuyas, estrato medio, $05^{\circ} 10^{\prime} 51^{\prime \prime} \mathrm{S}-80^{\circ} 37^{\prime} 31^{\prime \prime} \mathrm{O}, 2,433 \mathrm{msnm}$, 16-VI-2014, colecta manual, G. Juárez.

Comentario. Mencionada como Macrolampis sp en Juárez \& González (2016a). El género Macrolampis fue sinonimizado recientemente con el género Photinus (Zaragoza-Caballero et al., 2020).

54. Photinus sin identificar sp. 2. +

Material examinado. 1 o, Piura, Ayabaca, Bosque de neblina de Cuyas, estrato medio, $05^{\circ} 10^{\prime} 51^{\prime \prime} \mathrm{S}-80^{\circ} 37^{\prime} 31^{\prime \prime} \mathrm{O}, 2,559 \mathrm{msnm}$, 16-XI-2018, colecta manual, G. Juárez.

Familia Cantharidae Imhoff, 1856

Subfamilia Silinae Mulsant, 1862

Género Discodon Gorham, 1881

55. Discodon sin identificar sp. 1.

Material examinado. 1 , Piura, Ayabaca, Bosque de neblina de Cuyas, estrato medio, $05^{\circ} 10^{\prime} 51^{\prime \prime} \mathrm{S}-80^{\circ} 37^{\prime} 31^{\prime \prime} \mathrm{O}, 2,433 \mathrm{msnm}$, 11-VII-2018, colecta manual, G. Juárez.

Comentario. Hasta el momento es el único individuo colectado del género Discodon en la región Piura.

Familia Dermestidae Latreille, 1804

Subfamilia Dermestinae Latreille, 1804

Género Dermestes Linnaeus, 1758

56. Dermestes (Dermestes) peruvianus Laporte, 1840

Material examinado. 1 \% , Piura, Ayabaca, Bosque de neblina de Cuyas, estrato bajo, $05^{\circ} 10^{\prime} 51^{\prime \prime} \mathrm{S}-80^{\circ} 37^{\prime} 31^{\prime \prime} \mathrm{O}, 2,120 \mathrm{msnm}, 11$ VII-2018, colecta manual, G. Juárez.

Comentario. Especie de amplia distribución, registrándose para Argentina, Bolivia, Chile, México, Estados Unidos, Europa y Perú (Ciro-Díaz et al., 2008). Para la región Piura se cita en bosques secos interandinos y bosques montanos de las provincias de Ayabaca y Huancabamba (Juárez-Noé \& González-Coronado, 2019). Los individuos examinados se colectaron sobre cueros secos de ganado vacuno.

Subfamilia Attageninae Laporte, $1840+$

Género Attagenus Latreille, $1802+$

57. Attagenus (Attagenus) fasciatus (Thunberg, 1795) + 
Material examinado. 2 우, Piura, Ayabaca, Bosque de neblina de Cuyas, estrato medio, $05^{\circ} 10^{\prime} 51^{\prime \prime} \mathrm{S}-80^{\circ} 37^{\prime} 31^{\prime \prime} \mathrm{O}, 2,431 \mathrm{msnm}$, 11-VII-2018, colecta manual, U. González.

Comentario. Especie de amplia distribución en la región Piura (Juárez-Noé \& González-Coronado, 2019). Los individuos examinados se colectaron en piel seca de caballo.

Familia Melyridae Leach, 1815

Subfamilia Melyrinae Leach, 1815

Género Astylus Laporte de Castelnau, 1836

58. Astylus longulus Constantin, 2011

Material examinado. $1 \hat{\sigma}$, Piura, Ayabaca, Bosque de neblina de Cuyas, estrato medio, $05^{\circ} 10^{\prime} 51^{\prime \prime} \mathrm{S}-80^{\circ} 37^{\prime} 31^{\prime \prime} \mathrm{O}, 2,350 \mathrm{msnm}$, 08-III-2018, colecta manual, G. Juárez.

Comentario. Especie que se distribuye en zonas andinas de Ecuador y Perú (Constantin, 2011; Juárez \& González, 2015). Para la región Piura se cita en bosques montanos de la provincia de Ayabaca (Juárez \& González, 2015; Juárez-Noé \& González-Coronado, 2019).

\section{Astylus lojaensis Constantin, 2011}

Material examinado. $1 \hat{\delta}$, Piura, Ayabaca, Bosque de neblina de Cuyas, estrato medio, $05^{\circ} 10^{\prime} 51^{\prime \prime} \mathrm{S}-80^{\circ} 37^{\prime} 31^{\prime \prime} \mathrm{O}, 2,398 \mathrm{msnm}$, 18-VI-2019, colecta manual, G. Juárez.

Comentario. Especie que se distribuye en zonas andinas de Ecuador y Perú (Constantin, 2011; Juárez \& González, 2015). Para la región Piura se cita en bosques montanos de la provincia de Ayabaca (Juárez \& González, 2015; Juárez-Noé \& González-Coronado, 2019).

60. Astylus bonplandi Erichson, $1847+$

Material examinado. $1 \hat{\delta}$, Piura, Ayabaca, Bosque de neblina de Cuyas, estrato medio, $05^{\circ} 10^{\prime} 51^{\prime \prime} \mathrm{S}-80^{\circ} 37^{\prime} 31^{\prime \prime} \mathrm{O}, 2,456 \mathrm{msnm}$, 18-XII-2019, colecta manual, G. Juárez.

Comentario. Especie que se distribuye en Colombia, Ecuador y Perú en zonas altoandinas de la cordillera de los Andes (Constantin, 2011). Para la región Piura se cita para bosques montanos de la provincia de Huancabamba (Juárez-Noé \& González-Coronado, 2019), con los datos presentados amplía su distribución dentro de la región a la provincia de Ayabaca.

Familia Erotylidae Latreille, $1802+$

Subfamilia Erotylinae Latreille, 1802

Género Mycotretus Lacordaire, $1802+$

61. Mycotretus $\sin$ identificar sp. 1.+

Material examinado. $1 \hat{\sigma}$, Piura, Ayabaca, Bosque de neblina de Cuyas, estrato medio, $05^{\circ} 10^{\prime} 51^{\prime \prime} \mathrm{S}-80^{\circ} 37^{\prime} 31^{\prime \prime} \mathrm{O}, 2,456 \mathrm{msnm}$, 18-XII-2019, colecta manual, G. Juárez.

COMENTARIO. Hasta el momento es el único individuo colectado del género Mycotretus en la región Piura.

Familia Coccinellidae Latreille, 1807

Subfamilia Coccinellinae Latreille, 1807

Género Eriopis Mulsant, 1850

62. Eriopis sin identificar sp. 1

Material examinado. 1 $\widehat{\jmath}$, Piura, Ayabaca, Bosque de neblina de Cuyas, estrato medio, $05^{\circ} 10^{\prime} 51^{\prime \prime} \mathrm{S}-80^{\circ} 37^{\prime} 31^{\prime \prime} \mathrm{O}, 2,501 \mathrm{msnm}$, 28-III-2018, colecta manual, G. Juárez.

COMENTARIo. Hasta el momento es el único individuo colectado del género Eriopis en la región Piura.

Género Neda Mulsant, 1850

63. Neda aequatoriana Mulsant, 1853 (Fig. 11)

Material examinado. $1 \hat{\delta}$, Piura, Ayabaca, Bosque de neblina de Cuyas, estrato medio, $05^{\circ} 10^{\prime} 51^{\prime \prime} \mathrm{S}-80^{\circ} 37^{\prime} 31^{\prime \prime} \mathrm{O}, 2,689 \mathrm{msnm}$, 07-X-2019, colecta manual, G. Juárez.
Comentario. Especie de zonas andinas que se distribuye en Colombia, Ecuador, Venezuela y Perú (González, 2007). Para la región Piura se cita en bosques secos interandinos y bosques montanos de las provincias de Ayabaca y Huancabamba (Juárez-Noé \& González-Coronado, 2019).

Género Epilachna Chevrolat, 1837

64. Epilachna flavofasciata (Laporte, 1840) (Fig. 12)

Material examinado. $1 \hat{\sigma}$, Piura, Ayabaca, Bosque de neblina de Cuyas, estrato medio, $05^{\circ} 10^{\prime} 51^{\prime \prime} \mathrm{S}-80^{\circ} 37^{\prime} 31^{\prime \prime} \mathrm{O}, 2,689 \mathrm{msnm}, 22-$ VII-2018, colecta manual, G. Juárez; 1 đ̊, Piura, Ayabaca, Bosque de neblina de Cuyas, estrato alto, $05^{\circ} 10^{\prime} 51^{\prime \prime} \mathrm{S}-80^{\circ} 37^{\prime} 31^{\prime \prime} \mathrm{O}, 2,734$ msnm, 07-XII-2019, colecta manual, G. Juárez.

Comentario. Especie que se registra en bosques amazónicos y montanos de Colombia, Ecuador, Venezuela y Perú (González, 2007, 2015). Para la región Piura se cita en bosques montanos de la provincia de Ayabaca (Juárez-Noé \& González-Coronado, 2019).

65. Epilachna ambigua Mader, 1958 (Fig. 12)

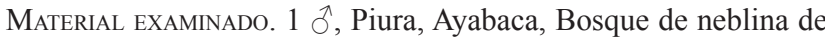
Cuyas, estrato alto, $05^{\circ} 10^{\prime} 51^{\prime \prime} \mathrm{S}-80^{\circ} 37^{\prime} 31^{\prime \prime} \mathrm{O}, 2,767 \mathrm{msnm}, 21$ VII-2018, colecta manual, G. Juárez.

Comentario. Especie que se distribuye en zonas andinas de Bolivia y Perú (Gordon, 1975; González, 2007; Juárez, 2014). Para Perú se cita solamente de la región Piura en bosques húmedos de montaña de la provincia de Ayabaca (Juárez, 2014; JuárezNoé \& González-Coronado, 2019).

66. Epilachna viridilineata Crotch, $1874+$

Material examinado. 1 $\approx$, Piura, Ayabaca, Bosque de neblina de Cuyas, estrato alto, $05^{\circ} 10^{\prime} 51^{\prime \prime} \mathrm{S}-80^{\circ} 37^{\prime} 31^{\prime \prime} \mathrm{O}, 2,732 \mathrm{msnm}, 21$ IV-2019, colecta manual, G. Juárez.

COMENTARIo. Especie que se distribuye en zonas andinas de Ecuador y Perú (Gordon, 1975; González, 2007, 2015). Para Perú se cita solamente de la región Piura en bosques húmedos de montaña de las provincias de Morropón y Ayabaca (González, 2007; Juárez-Noé \& González-Coronado, 2019).

67. Epilachna discolor Erichson, $1847+$

Material eXAminado. $1 \hat{\delta}$, Piura, Ayabaca, Bosque de neblina de Cuyas, estrato alto, $05^{\circ} 10^{\prime} 51^{\prime \prime} \mathrm{S}-80^{\circ} 37^{\prime} 31^{\prime \prime} \mathrm{O}, 2,745 \mathrm{msnm}, 20$ IV-2019, colecta manual, G. Juárez.

COMENTARIO. Especie que se distribuye en zonas andinas de Bolivia y Perú (Gordon, 1975; González, 2007, 2015). En la región Piura se registra en bosques húmedos de montaña de la provincia de Ayabaca (Juárez-Noé \& González-Coronado, 2019).

Familia Meloidae Gyllenhal, 1810

Subfamilia Meloinae Gyllenhal, 1810

Género Lyttamorpha Kaszab, 1959

68. Lyttamorpha reichenbachi Kirsch, 1866

Material examinado. 1 $\widehat{\jmath}$, Piura, Ayabaca, Bosque de neblina de Cuyas, estrato alto, $05^{\circ} 10^{\prime} 51^{\prime \prime} \mathrm{S}-80^{\circ} 37^{\prime} 31^{\prime \prime} \mathrm{O}, 2,745 \mathrm{msnm}, 11-$ I-2018, colecta manual, G. Juárez.

Comentario. Especie que se distribuye en Argentina, Colombia, Ecuador y Perú (regiones de Piura y Cusco) en zonas altoandinas (Pinto \& Bologna, 2016; Juárez-Noé \& González-Coronado, 2019). En la región Piura se cita para bosques montanos de la provincia de Ayabaca (Juárez-Noé \& González-Coronado, 2019).

Familia Cerambycidae Latreille, 1802

Subfamilia Prioninae Latreille, 1802

Género Prionacalus White, 1845

69. Prionacalus cacicus (White, 1845) (Fig. 13)

Material eXaminado. $2 \delta^{\lambda}$, Piura, Ayabaca, Bosque de neblina de Cuyas, estrato alto, $05^{\circ} 10^{\prime} 51^{\prime \prime} \mathrm{S}-80^{\circ} 37^{\prime} 31^{\prime \prime} \mathrm{O}, 2,712 \mathrm{msnm}, 12$ - 


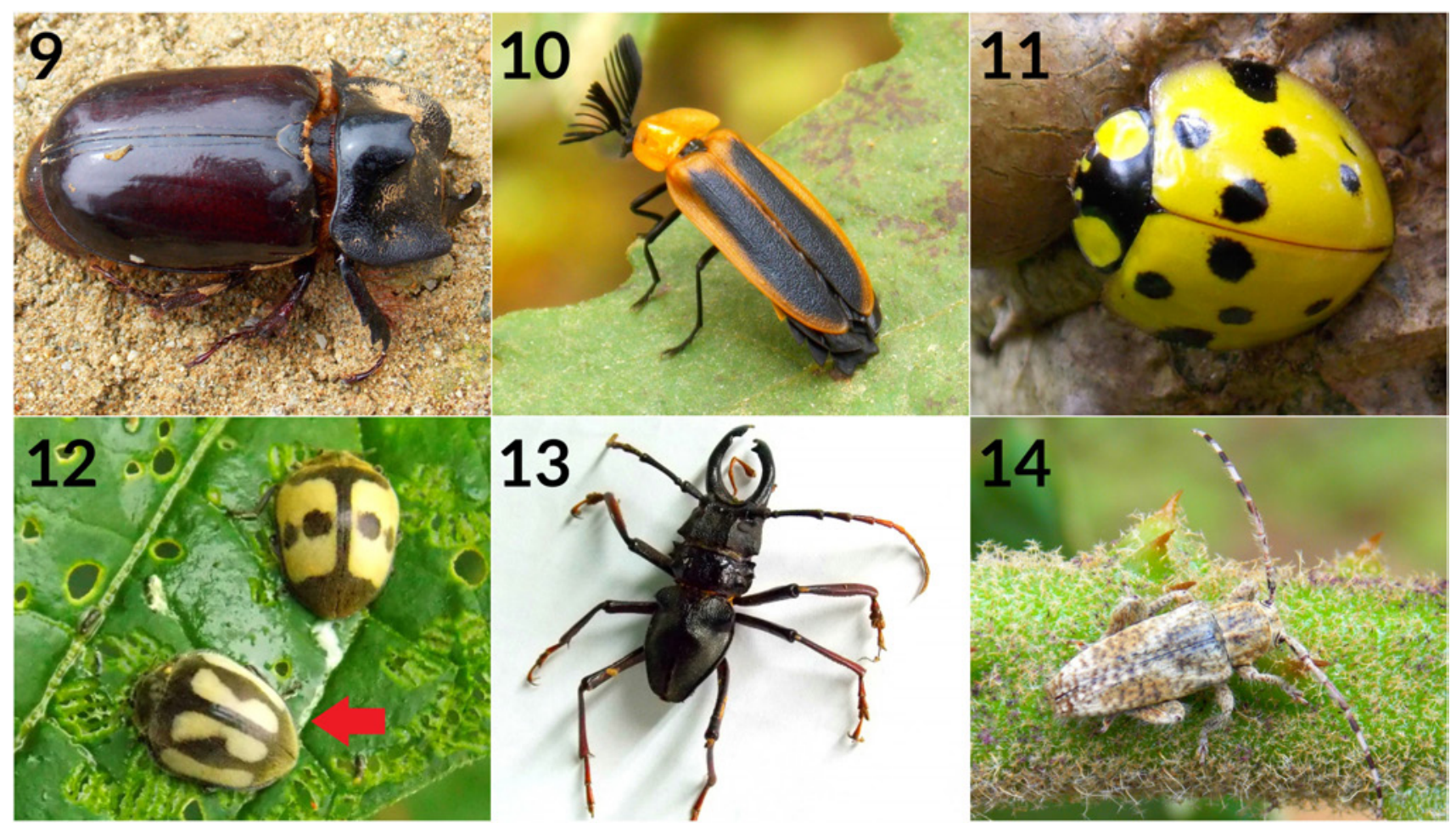

Figs. 9-14.- Habitus. 9. Heterogomphus rubripennis Prell, 1912. 10. Cladodes sin identificar sp. 1. 11. Neda aequatoriana Mulsant, 1853. 12. Epilachna flavofasciata (Laporte, 1840); Epilachna ambigua Mader, 1958 (flecha roja). 13. Prionacalus cacicus (White, 1845). 14. Bisaltes (Bisaltes) fuscomarmoratus Breuning, 1966. Fotografías por G. Juárez and U. González.

Figs. 9-14.- Habitus. 9. Heterogomphus rubripennis Prell, 1912. 10. Cladodes sin identificar sp. 1. 11. Neda aequatoriana Mulsant, 1853. 12. Epilachna flavofasciata (Laporte, 1840); Epilachna ambigua Mader, 1958 (red arrow). 13. Prionacalus cacicus (White, 1845). 14. Bisaltes (Bisaltes) fuscomarmoratus Breuning, 1966. Photographs by G. Juárez and U. González.

I-2018, colecta manual, G. Juárez; 1 §̂, Piura, Ayabaca, Bosque de neblina de Cuyas, estrato medio, $05^{\circ} 10^{\prime} 51^{\prime \prime} \mathrm{S}-80^{\circ} 37^{\prime} 31^{\prime \prime} \mathrm{O}$, 2,606 msnm, 11-VI-2019, colecta manual, U. González.

Comentario. Especie que se distribuye en Colombia, Ecuador y Perú (Santos-Silva et al., 2013). En la región Piura se cita para las provincias de Ayabaca y Huancabamba en zonas andinas a partir de los 2,000 msnm (Juárez \& González, 2016b; JuárezNoé \& González-Coronado, 2019).

Género Psalidognathus Gray, 1831

70. Psalidognathus antonkozlovi Noguchi \& Santos-Silva, 2016 *

Material examinado. 1 $\delta$, Piura, Ayabaca, Bosque de neblina de Cuyas, estrato medio, $05^{\circ} 10^{\prime} 51^{\prime \prime} \mathrm{S}-80^{\circ} 37^{\prime} 31^{\prime \prime} \mathrm{O}, 2,630 \mathrm{msnm}$, 12-V-2016, colecta manual, G. Juárez, 066 CER-GJN. 1 ô, Piura, Ayabaca, Bosque de neblina de Cuyas, estrato alto, $05^{\circ} 10^{\prime} 51^{\prime \prime} \mathrm{S}-80^{\circ} 37^{\prime} 31^{\prime \prime} \mathrm{O}, 2,715 \mathrm{msnm}, 12-\mathrm{X}-2018$, colecta manual, G. Juárez.

Comentario. Especie citada como Psalidognathus sp en Juárez \& González (2016a). Especie conocida únicamente en Perú desde la región Piura en zonas andinas de la provincia de Huancabamba (Noguchi \& Santos-Silva, 2016; Juárez-Noé \& González-Coronado, 2019). Con los datos presentados aquí amplía su distribución a la provincia de Ayabaca.

Subfamilia Lamiinae Latreille, 1825

Género Bisaltes Thomson, 1860

71. Bisaltes (Bisaltes) fuscomarmoratus Breuning, 1966 (Fig. 14)

Material examinado. 1 $\widehat{\jmath}$, Piura, Ayabaca, Bosque de neblina de Cuyas, estrato medio, $05^{\circ} 10^{\prime} 51^{\prime \prime} \mathrm{S}-80^{\circ} 37^{\prime} 31^{\prime \prime} \mathrm{O}, 2,521 \mathrm{msnm}$, 12-VI-2016, colecta manual, G. Juárez, 069 CER-GJN; 1 \$ే,
Piura, Ayabaca, Bosque de neblina de Cuyas, estrato alto, $05^{\circ} 10^{\prime} 51^{\prime \prime} \mathrm{S}-80^{\circ} 37^{\prime} 31^{\prime \prime} \mathrm{O}, 2,722 \mathrm{msnm}, 23-\mathrm{IX}-2019$, colecta manual, U. González.

Comentario. Especie citada como Bisaltes sp. en Juárez \& González (2016a). Para la región Piura se distribuye en zonas andinas de la provincia de Ayabaca (Juárez-Noé \& GonzálezCoronado, 2019).

Género Fredlanea Martins \& Galileo, 1996

72. Fredlanea wilderi Juárez-Noé \& González-Coronado, 2020 * (Fig. 15)

Material examinado. 1 , Piura, Ayabaca, Bosque de neblina de Cuyas, estrato medio, $05^{\circ} 10^{\prime} 51^{\prime \prime} \mathrm{S}-80^{\circ} 37^{\prime} 31^{\prime \prime} \mathrm{O}, 2,634 \mathrm{msnm}$, 14-VI-2016, colecta manual, G. Juárez, 072 CER-GJN.

Comentario. Especie citada como Fredlanea sp. en Juárez \& González (2016a). Especie conocida únicamente en Perú desde la región Piura en bosques montanos de la provincia de Ayabaca (Juárez-Noé \& González-Coronado, 2020).

Género Taeniotes Audinet-Serville, $1835+$

73. Taeniotes marmoratus Thomson, $1865+$ (Fig. 16)

Material eXaminado. 1 ㅇ, Piura, Ayabaca, Bosque de neblina de Cuyas, estrato medio, $05^{\circ} 10^{\prime} 51^{\prime \prime} \mathrm{S}-80^{\circ} 37^{\prime} 31^{\prime \prime} \mathrm{O}, 2,511 \mathrm{msnm}$, 14-IX-2019, colecta manual, G. Juárez.

Comentario. Especie que se distribuye en Ecuador y Perú (Monné, 2019; Juárez-Noé \& González-Coronado, 2019). Para la región Piura se cita en bosques montanos de la provincia de Ayabaca (Juárez-Noé \& González-Coronado, 2019).

Familia Chrysomelidae Latreille, 1802

Subfamilia Cassidinae Gyllenhal, 1813 
Género Sceloenopla Chevrolat, 1837

74. Sceloenopla annulipes Pic, 1932

Material examinado. 1 , Piura, Ayabaca, Bosque de neblina de Cuyas, estrato medio, $05^{\circ} 10^{\prime} 51^{\prime \prime} \mathrm{S}-80^{\circ} 37^{\prime} 31^{\prime \prime} \mathrm{O}, 2,511 \mathrm{msnm}$, 15-IX-2019, colecta manual, G. Juárez.

Comentario. Especie citada como Sceloenopla sp. en Juárez \& González (2016a). En Perú esta especie se distribuye en las regiones de Cusco y Piura (Juárez-Noé \& González-Coronado, 2019).

Subfamilia Galerucinae Latreille, 1802

Género Metalepta Baly, $1861+$

75. Metalepta $\sin$ identificar sp. 1. +

Material examinado. 1 ㅇ, Piura, Ayabaca, Bosque de neblina de Cuyas, estrato medio, $05^{\circ} 10^{\prime} 51^{\prime \prime} \mathrm{S}-80^{\circ} 37^{\prime} 31^{\prime \prime} \mathrm{O}, 2,569 \mathrm{msnm}$, 02-VI-2019, colecta manual, G. Juárez.

COMEnTARIO. El género se distribuye en zonas andinas de Ecuador y Perú (Beenen, 2013; Juárez-Noé \& González-Coronado, 2019). El individuo examinado fue colectado a ras del suelo sobre la hojarasca.

Género Diabrotica Chevrolat, 1837

76. Diabrotica mauliki Barber, 1947

Material examinado. 1 , Piura, Ayabaca, Bosque de neblina de Cuyas, estrato medio, $05^{\circ} 10^{\prime} 51^{\prime \prime} \mathrm{S}-80^{\circ} 37^{\prime} 31^{\prime \prime} \mathrm{O}, 2,632 \mathrm{msnm}$, 03-VI-2016, colecta manual, G. Juárez, 073 CHR-GJN; 1 ㅇ, Piura, Ayabaca, Bosque de neblina de Cuyas, estrato medio, $05^{\circ} 10^{\prime} 51^{\prime \prime} \mathrm{S}-80^{\circ} 37^{\prime} 31^{\prime \prime} \mathrm{O}, 2,409$ msnm, 013-XI-2019, colecta manual, G. Juárez.

Comentario. Especie citada como Diabrotica sp. en Juárez \& González (2016a).

\section{Diabrotica $\mathrm{cf}$. lucifera Erichson, $1847+$}

Material examinado. 1 , Piura, Ayabaca, Bosque de neblina de Cuyas, estrato medio, $05^{\circ} 10^{\prime} 51^{\prime \prime} \mathrm{S}-80^{\circ} 37^{\prime} 31^{\prime \prime} \mathrm{O}, 2,521 \mathrm{msnm}$, 03-VI-2018, colecta manual, G. Juárez.

Subfamilia Alticinae Latreille, 1802

Género Altica Fabricius, 1775

78. Altica sin identificar sp. 1.

Material examinado. $2 \hat{\partial} \hat{\partial}, 1$, Piura, Ayabaca, Bosque de neblina de Cuyas, estrato bajo, $4^{\circ} 37^{\prime} \mathrm{S}-79^{\circ} 45^{\prime} \mathrm{O}, 2,023$

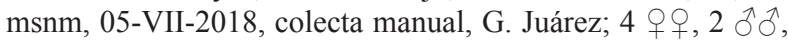
Piura, Ayabaca, Bosque de neblina de Cuyas, estrato medio, $4^{\circ} 33^{\prime} \mathrm{S}-79^{\circ} 46^{\prime} \mathrm{O}, 2,600 \mathrm{msnm}, 02-\mathrm{X}-2019$, colecta manual, U. González; 2 $\hat{\jmath}, 2$ 우, Piura, Ayabaca, Bosque de neblina de Cuyas, estrato alto, $4^{\circ} 38^{\prime} \mathrm{S}-7^{\circ} 47^{\prime} \mathrm{O}, 2,789 \mathrm{msnm}, 12-\mathrm{XI}-$ 2019, colecta manual, G. Juárez.

Comentario. La mayoría de los individuos se colectaron sobre hojas de los géneros Baccharis L. (Asteraceae) y Myrsine L. (Myrsinaceae), mientras que otros se colectaron a ras de suelo.

Género Asphaera Chevrolat, 1843

79. Asphaera albomarginata (Latreille, 1833) (Fig. 17)

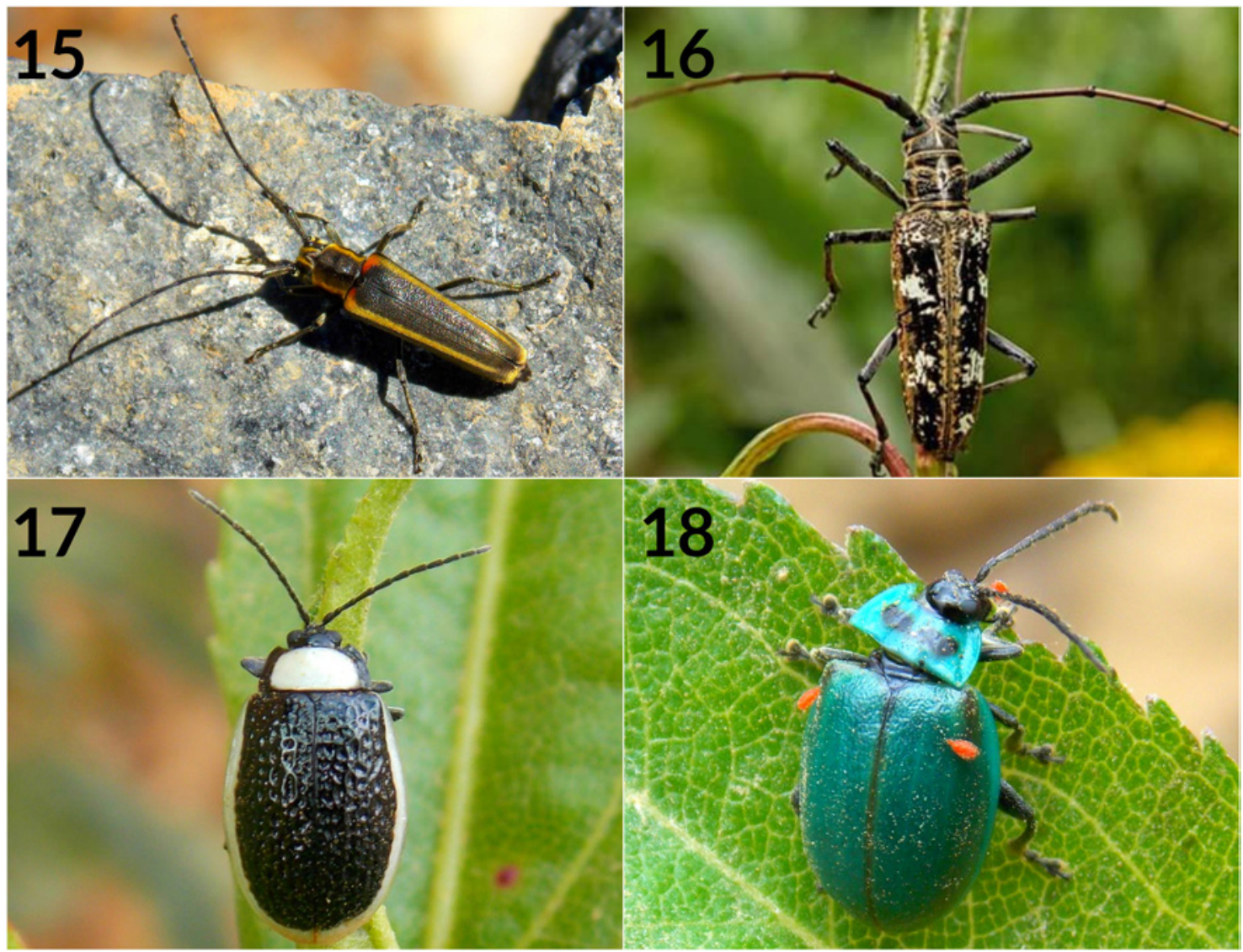

Figs. 15-18.- Habitus. 15. Fredlanea wilderi Juárez-Noé \& González-Coronado, 2020. 16. Taeniotes marmoratus Thomson, 1865. 17. Asphaera albomarginata (Latreille, 1833). 18. Aspicela nigroviridis Guerin, 1855. Fotografías 15, 17-18 por G. Juárez y U. González; fotografía 16 por I. Saldaña.

Figs. 15-18.- Habitus. 15. Fredlanea wilderi Juárez-Noé \& González-Coronado, 2020. 16. Taeniotes marmoratus Thomson, 1865. 17. Asphaera albomarginata (Latreille, 1833). 18. Aspicela nigroviridis Guerin, 1855. Photographs $15,17-18$ by G. Juárez and U. González; photography 16 by I. Saldaña. 
Material examinado. $2 \hat{\jmath} \widehat{\partial}, 2$ $q \propto$, Piura, Ayabaca, Bosque de neblina de Cuyas, estrato bajo, $4^{\circ} 37^{\prime} \mathrm{S}-79^{\circ} 45^{\prime} \mathrm{O}, 2,003 \mathrm{msnm}$, 05-VII-2018, colecta manual, G. Juárez; 1 , Piura, Ayabaca, Bosque de neblina de Cuyas, estrato medio, $4^{\circ} 33^{\prime} \mathrm{S}-79^{\circ} 46^{\prime} \mathrm{O}$, 2,600 msnm, 02-X-2018, colecta manual, U. González; 3 กิ ภે, 2 우, Piura, Ayabaca, Bosque de neblina de Cuyas, estrato alto, $4^{\circ} 38^{\prime} \mathrm{S}-79^{\circ} 47^{\prime} \mathrm{O}, 2789$ msnm, 12-XI-2018, colecta manual, G. Juárez; 1 đ̊, 2 오, Piura, Ayabaca, Bosque de neblina de Cuyas, estrato bajo, 43' ${ }^{\prime} \mathrm{S}-79^{\circ} 43^{\prime} \mathrm{O}, 2,200 \mathrm{msnm}$, 23-II-2019, colecta manual, U. González; 1 స̃, 1 †, Piura, Ayabaca, Bosque de neblina de Cuyas, estrato medio, $4^{\circ} 35^{\prime} \mathrm{S}-79^{\circ} 43^{\prime} \mathrm{O}, 2,434 \mathrm{msnm}$, 10-VII-2019, colecta manual, G. Juárez; $2 \overbrace{}^{\lambda}$, Piura, Ayabaca, Bosque de neblina de Cuyas, estrato alto, $4^{\circ} 32^{\prime} \mathrm{S}-79^{\circ} 40^{\prime} \mathrm{O}$, 2,900 msnm, 23-II-2019, colecta manual, G. Juárez.

Comentario. Especie que se distribuye a partir de bosques secos interandinos y bosque húmedos de montaña de la región Piura (Juárez-Noé \& González-Coronado, 2018, 2019). La mayoría de los individuos se colectaron sobre hojas de los géneros Baccharis L. (Asteraceae) y Myrsine L. (Myrsinaceae), mientras que otros se colectaron cerca del suelo sobre la vegetación circundante.

Género Aspicela Dejean, 1837

80. Aspicela nigroviridis Guerin, 1855 (Fig. 18)

Material examinado. $2 \hat{\jmath} \partial^{\lambda}$, Piura, Ayabaca, Bosque de neblina de Cuyas, estrato bajo, 4 $37^{\prime} \mathrm{S}-79^{\circ} 45^{\prime} \mathrm{O}, 2,103 \mathrm{msnm}$, 11-III2018, colecta manual, G. Juárez; 2 స̂, 1 +, Piura, Ayabaca, Bosque de neblina de Cuyas, estrato medio, $4^{\circ} 33^{\prime} \mathrm{S}-79^{\circ} 46^{\prime} \mathrm{O}$,

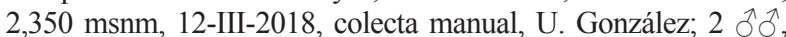
1 ㅇ, Piura, Ayabaca, Bosque de neblina de Cuyas, estrato alto, $4^{\circ} 38^{\prime} \mathrm{S}-79^{\circ} 47^{\prime} \mathrm{O}, 2,789 \mathrm{msnm}, 12-\mathrm{XI}-2018$, colecta manual, U. González; 1 , Piura, Ayabaca, Bosque de neblina de Cuyas, estrato bajo, 4 $36^{\prime} \mathrm{S}-79^{\circ} 43^{\prime} \mathrm{O}, 2,156 \mathrm{msnm}, 23-\mathrm{II}-2019$, colecta manual, G. Juárez; 2 ภิ $\widehat{0}, 2$ 우, Piura, Ayabaca, Bosque de neblina de Cuyas, estrato medio, $4^{\circ} 35^{\prime} \mathrm{S}-79^{\circ} 43^{\prime} \mathrm{O}, 2,531 \mathrm{msnm}, 10-\mathrm{IV}$ -

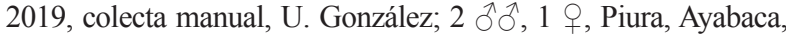
Bosque de neblina de Cuyas, estrato alto, $4^{\circ} 32^{\prime} \mathrm{S}-79^{\circ} 40^{\prime} \mathrm{O}$, 2,899 msnm, 12-IX-2019, colecta manual, U. González.

Comentario. Especie que se distribuye en zonas andinas de Colombia (Bechyné \& Bechyné, 1971) y Perú (provincias de Huancabamba y Ayabaca de la región Piura) (Juárez \& González, 2016c; Juárez-Noé \& González-Coronado, 2019). Los individuos se colectaron sobre hojas de los géneros Baccharis L. (Asteraceae) y Myrsine L. (Myrsinaceae).

Familia Curculionidae Latreille, 1802

Subfamilia Entiminae Schönherr, 1833

Género Brachyomus Lacordaire, 1863

81. Brachyomus sin identificar sp. 1.

Material examinado. $1 \hat{\jmath}$, Piura, Ayabaca, Bosque de neblina de Cuyas, estrato bajo, $4^{\circ} 32^{\prime} \mathrm{S}-79^{\circ} 40^{\prime} \mathrm{O}, 2,103 \mathrm{msnm}, 25-\mathrm{IV}-206$, colecta manual, G. Juárez., 105 CUR-GJN.

Comentario. Citado como Compsus sp en Juárez \& González (2016a). El individuo fue colectado a ras del suelo desprovisto de vegetación.

\section{Agradecimientos}

Al señor Esteban Aguilera del caserío Yacupampa por brindarnos todas las facilidades necesarias para el ingreso al bosque de Cuyas y acompañarnos como guía en las diferentes zonas de muestreo. A los entomólogos Carlos A. Julio (Carabidae, Scarabaeidae), Guillermo González (Coccinellidae), Rob Westerduijn, Michael Geiser, Wills Flowers y Charles Staines (Chrysomelidae), Angélico Asenjo (Staphylinidae), Miguel Monné (Cerambycidae),
Paul Johnson (Elateridae) y Cesar Neita (Scarabaeidae: Dynastinae) por su ayuda en la identificación de algunos especímenes y por el envío de material bibliográfico.

\section{Referencias}

Abadie, E., Grossi. P. \& Wagner, P., 2008. A field guide of the Dynastinae family of the South America. Argentina. 119 pp.

Ambulay, M., 2006. Etnobotánica en las comunidades campesinas Cuyas-Cuchayo, Joras y Suyupampa del Bosque de Cuyas, Ayabaca-Piura. Tesis para optar el título profesional de biólogo. Universidad Nacional de Piura. Perú.

Archangelsky, M., Manzo, V., Michat, M. C. \& Torres, P. L. M., 2009. Coleoptera. En: E. Domínguez \& H. R. Fernández (eds.). Macroinvertebrados bentónicos sudamericanos. Sistemática y Biología. Fundación Miguel Lillo, Tucumán-Argentina: 411-468.

Arias-Buriticá, J. A., 2011. Revisión taxonómica de la Sección "Buqueti", Dichotomius Hope, 1838 (Coleoptera: Scarabaeidae: Scarabaeinae). Tesis para optar título de Magister en Ciencias Biológicas. Universidad Nacional de Colombia.

Bachmann, A., 1966. El grupo Stethoxus insularis (Coleoptera, Hydrophilidae). Revista de la Sociedad Entomologica Argentina, 28(1-4): 9-16.

Balthasar, V., 1942. Neue Scarabaeiden aus Süd-Amerika. 72. Beitrag zur Kenntnis der Scarabaeiden. (Col.). Casopis Ceskoslovenske Spolecnosti Entomologicke, 39: 36-44.

Bartolozzi, 1. \& Onore, G., 2006. Sphaenognathus (Chiasognathinus) xerophilus sp.n. from Peru (Coleoptera: Lucanidae). Koleopterologische Rundschau, 76: 361365.

Bechyné, J. \& Bechyné, B., 1971. Catalogue of Neotropical Alticinae. Copia no publicada depositada en el Museo del Instituto de Zoología Agrícola, Universidad Central de Venezuela, Maracay.

Beenen, R., 2013. New species and a new genus of brachelytrous Galerucinae (Coleoptera: Chrysomelidae). Entomologische Zeitschrift, 123(4): 173-183.

Benetti, C., Régil. J. \& Garrido, J., 2003. Estudio faunístico de Hydradephaga (Coleoptera: Dytiscidae, Gyrinidae, Haliplidae, Noteridae) en el municipio de Gramado, Sur de Brasil. Boletín de la Sociedad Entomológica Aragonesa (S.E.A.), 32: 37-44.

Blanco-Aller, R., 2016. Contribución al conocimiento de los géneros Megadytes Sharp, 1882, Thermonectus Dejean, 1833 e Hydaticus Leach, 1817 (Coleoptera: Adephaga: Dytiscidae) en Costa Rica. Boletín de la Sociedad Entomológica Aragonesa (S.E.A.), 58: 197-205.

Bocákova, M., 2010. Revision of the Tribe Calopterini (Coleoptera, Lycidae). Studies on Neotropical Fauna and Environment, 38: 207-234.

Bouchard, P., Bousquet, Y., Davies, A., Alonso-Zarazaga, M.A., Lawrence, J., Lyal, C., Newton, A., Reid, C., Schmitt, M., Slipinski, A. \& Smith, A., 2011. Family-group names in Coleoptera (Insecta). ZooKeys, 88: 1-972. https://doi.org/10.3897/zookeys.88.807 
Breuning, S., 1966. Nouvelles formes de Lamiaires (Dix-Septième Partie). Bulletin Institut Royal des Sciences Naturelles de Belgique, 42(21): 1-24.

Bustamante-Navarrete, A., Oros-Ramos, A., Yábar-Landa, E., Marquina-Montesinos, E. L. \& Elme-Tumpay, A., 2017. Contribución al conocimiento de los escarabajos de la familia Silphidae (Coleoptera) en el Perú. Arquivos Entomolóxicos, 17: 135-143.

Bustamante, A., 2018. Registro de los coleópteros acuáticos en la región Cusco, Perú. The Biologist (Lima), 16(1): 39-49.

Chaboo, C., 2015. Beetles (Coleoptera) of Peru: A Survey of the Families. Part I. Overview. Journal of the Kansas Entomological Society, 88(2): 135-139. https://doi. org/10.2317/0022-8567-88.2.135

Chani-Posse, M., 2011. Redescription of some South American species of Belonuchus (Coleoptera: Staphylinidae), with a checklist of the southern South American species of the genus. Revista de la Sociedad Entomológica Argentina, 70(3-4): 213-229.

Ciro-Díaz, W. Anteparra, M.E. \& Hermann, A., 2008. Dermestidae (Coleoptera) en el Perú: revisión y nuevos registros. Revista Peruana de Biología, 15(1): 15-20. https://doi.org/10.15381/rpb.v15i1.1662

Constantin, R., 2011. A contribution to the genus Astylus Laporte de Castelnau, 1836 in Ecuador, with descriptions of three new species (Coleoptera, Melyridae). Entomologica Basiliensia, 33: 39-61.

Cook, J., 2002. A revision of the Neotropical genus Cryptocanthon Balthasar (Coleoptera: Scarabaeidae: Scarabaeinae). The Coleopterists Bulletin, 56(mo1): 3-96. https://doi.org/10.1649/0010-065X(2002)56[3:A ROTNG]2.0.CO;2

Dillon, L. S. \& Dillon, E. S., 1941. The tribe Monochamini in the Western Hemisphere (Coleoptera: Cerambycidae). Scientific Publications of the Reading Public Museum, 1: 1-135. Disponible en: https://catalog.hathitrust. org/Record/001500648 [consultado 28 Oct. 2020].

Endrödi, S., 1985. The Dynastinae of the World. W. Junk Publisher. 842 pp.

Farfán, M., 2007. Fanerógamas del Bosque de Cuyas, Ayabaca. Tesis para optar el título profesional de Biólogo. Universidad Nacional de Piura. Piura, Perú.

Figueroa, 1. \& Ratcliffe, B., 2016. A new species of Ancognatha Erichson (Coleoptera: Scarabaeidae: Dynastinae: Cyclocephalini) from Peru, with distributions of Peruvian Ancognatha species. The Coleopterists Bulletin, 70(1): 65-72. https://doi.org/10.1649/072.070.0107

Flanagan, J. \& Vellinga, W., 2000. Tres bosques nublados de Ayabaca: su avifauna y conservación. ProAves Perú. 52 pp.

Furth, D. G. \& Suzuki, K., 1998. Studies on Oriental and Australian Alticinae genera, based on the comparative morphology of the metafemoral spring, genitalia, and hind wing venation. In: M. Biondi, M. Daccordi \& D. G. Furth (eds.). Proceedings of the Fourth International Symposium on the Chrysomelidae. Museo Regionale Scienze Naturali Torino: 1-124.

Furth, D., Savini, V. \& Chaboo, C., 2015. Beetles (Coleoptera) of Peru: a survey of the families. Chrysomelidae:
Alticinae (Flea Beetles). Journal of the Kansas Entomological Society, 88(3): 368-374. https://doi.org/10.2317/ kent-88-03-368-374.1

Gasca, H., Vasconcelos, R. \& Ratcliffe, B., 2008. Synopsis of the Oryctini (Coleoptera: Scarabaeidae: Dynastinae) from the Brazilian Amazon. Insecta Mundi, 0061: 1-62.

Génier, F., 1996. A revision of the Neotropical genus Ontherus Erichson (Coleoptera: Scarabaeidae, Scarabaeinae). Memoirs of the Entomological Society of Canada, 170: 1-173.

Génier, F. \& Kohlmann, B., 2003. Revision of the Neotropical dung beetle genera Scatimus Erichson and Scatrichus gen. nov. (Coleoptera: Scarabaeidae: Scarabaeinae). Fabreries, 28(2): 57-11.

Giraldo, A. \& Arellano, G., 2003. Resiliencia de la comunidad epígea de Coleoptera en las Lomas de Lachay después del evento El Niño 1997-98. Ecología Aplicada, 2(1): 59-68. Disponible en: http://www.lamolina. edu.pe/ecolapl/Resilencia_coleopteros_Lachay.htm [consultado 28 Oct. 2020].

González, G., 2015. Beetles (Coleoptera) of Peru: A survey of the Families. Coccinellidae. Journal of the Kansas Entomological Society, 88(2): 229-236. https://doi. org/10.2317/kent-88-02-229-236.1

González, G., 2007. Los Coccinellidae de Perú. Disponible en: https://www.coccinellidae.cl/paginasWebPeru/Paginas/InicioPeru.php [consultado 28 Oct. 2020].

Gordon, R., 1975. A revision of Epilachninae of the Western Hemisphere (Coleoptera: Coccinellidae). Technical Bulletin, 1493: 1-409.

Háva, J., 2004. World keys to the genera and subgenera of Dermestidae (Coleoptera), with descriptions, nomenclature and distributional records. Acta Musei Nationalis Pragae, Natural History, 60(3-4): 149-164.

Juárez, G., 2014. Cuatro nuevos registros de insectos en los bosques de la Región Piura, Perú. The Biologist (Lima), 12(2): 297-304.

Juárez, G. \& González, U., 2015. Dos nuevos registros del género Astylus Laporte de Castelnau, 1836 (Coleoptera: Melyridae) para Perú. The Biologist (Lima), 13(2): 271 277.

Juárez, G. \& González, U., 2016a. Coleópteros (Insecta: Coleoptera) del bosque de neblina de Cuyas, Ayabaca-Región Piura, Perú. The Biologist (Lima), 14(2): 199-217.

Juárez, G. \& González, U., 2016b. Nuevos registros de distribución de doce especies de Cerambycidae (Coleoptera) en Perú. Boletín de la Sociedad Entomológica Aragonesa (S.E.A.), 59: 263-266.

Juárez, G. \& González, U., 2016c. Primer registro de Aspicela nigroviridis Guerin, 1855 (Coleoptera: Chrysomelidae: Alticinae) para Peru. Arquivos Entomolóxicos, 15: 275-278.

Juárez-Noé, G. \& González-Coronado, U., 2018. Lista de coleópteros (Insecta: Coleoptera) de la región Piura, Perú. Folia Entomológica Mexicana (n.s.), 4(1): 1-27.

Juárez-Noé, G. \& González-Coronado, U., 2019. Actualización a la lista de coleópteros (Insecta: Coleoptera) de la región Piura, Perú. Graellsia, 75(2): e097. https://doi. org/10.3989/graellsia.2019.v75.233 
Juárez-Noé, G. \& González-Coronado, U., 2020. A new species of Fredlanea Martins \& Galileo, 1996 from Peruvian Andes (Coleoptera: Cerambycidae, Lamiinae, Hemilophini). Fragmenta Entomologica, 52(1): 49-52. https://doi.org/10.13133/2284-4880/406

Lachaume, G., 1985. Les coléoptères du Monde, Vol 5, Dynastini. 1. Dynastes-Megasoma-Golofa. Sciences Nat, Venette. $85 \mathrm{pp}$.

Ledo, A., 2012. On the Spatial Distribution of Woody Plant Species in a Tropical Montane Cloud Forest. Tesis Doctoral. Escuela Técnica Superior de Ingenieros de Montes. Universidad Politécnica de Madrid. Madrid, España.

Libonatti, M., Michat, M. \& Torres, P., 2011. Key to the subfamilies, tribes and genera of adult Dytiscidae of Argentina (Coleoptera: Adephaga). Revista de la Sociedad Entomológica Argentina, 70(3-4): 317-336.

Lozada, A., Fernández, I. \& Trujillo, M., 2004. Lista preliminar de los coleópteros (Insecta: Coleoptera) de Topes de Collantes, Trinidad, Sancti Spíritus, Cuba. Boletín de la Sociedad Entomológica Aragonesa (S.E.A.), 34: 101-106.

Martins, U. R. \& Galileo, M. H. M., 2014. Tribo Hemilophini Thomson, 1868. Parte II. In: U. R. Martins (ed.). Cerambycidae Sul-Americanos (Coleoptera). Taxonomia. Vol. 14. Sociedade Brasileira de Entomologia. Curitiba: 3-296.

Medina, C. A. \& Lopera-Toro, A., 2000. Clave ilustrada para la identificación de géneros de escarabajos coprófagos (Coleoptera: Scarabaeinae) de Colombia. Caldasia, 22(2): 299-315.

Ministerio Del Ambiente., 2018. Mapa Nacional de Ecosistemas del Perú. Memoria Descriptiva. Ministerio del Ambiente (MINAM). Lima-Perú. 60 pp.

Miller, K. \& Bergsten, L., 2016. Diving Beetles of the World. Johns Hopkins University Press. Baltimore. 320 pp.

Mohamedsaid, M. S. \& Furth, D. G., 2011. Secondary sexual characteristics in the Galerucinae (sensu stricto) (Coleoptera: Chrysomelidae). International Scholarly Research Network, Zoology, 328670: 1-60. https://doi. org/10.5402/2011/328670

Mondaca, J., 2012. Paranomala undulata peruviana (Scarabaeidae: Rutelinae): un nuevo escarabajo exótico introducido en Chile. Revista Chilena de Entomología, 37: $75-80$.

Monné, M., 2019. Catalogue of the Cerambycidae (Coleoptera) of the Neotropical Region. Part II. Subfamily Lamiinae. Disponible en: https://cerambycids.com/default. asp?action=show_catalog [consultado 28 Oct. 2020].

More, A., Villegas, P. \& Alzamora, M., 2014. Piura, Áreas prioritarias para la conservación de la biodiversidad. Naturaleza y Cultura Internacional-PROFONANPE. Lima. 166 pp.

Moret, P. \& Bousquet, Y., 1995. Le sous-genre Dercylus (Licinodercylus) Kuntzen, 1912: Position systemátique, revision des especes et description de la larve (Carabidae, Dercylini). The Canadian Entomologist, 127: 753-798.

Moret, P., 2005. Los coleópteros Carabidae del páramo en los Andes de Ecuador. Sistemática, ecología y biogeografía. Monografía 2. Museo de Zoología. Pontificia Universidad Católica de Ecuador. 154 pp.
Morón, M., 2003. Atlas de los escarabajos de México. Coleoptera: Lamellicornia. Vol II Familias Scarabaeidae, Trogidae, Passalidae y Lucanidae. Conabio, México D.F, México, 227 pp.

Morón, M. A., 2004. Escarabajos, 200 millones de años de evolución. Instituto de Ecología, A.C. y Sociedad Entomológica Aragonesa. Zaragoza. 204 pp.

Navarrete, J., Newton, A., Thayer, M., Ashe, J. \& Chandler, D., 2002. Guía ilustrada para los géneros de Staphylinidae (Coleoptera) de México. Comisión Nacional para el Conocimiento y Uso de la Biodiversidad. Universidad de Guadalajara y CONABIO, México. 404 pp.

Noguchi, T. \& Santos-Silva, A., 2016. A new species of Psalidognathus Gray, 1831 from Peru (Coleoptera: Cerambycidae: Prioninae). Insecta Mundi, 0508: 1-6.

Onore, G., Bartolozzi, L. \& Zilioli, M., 2011. A new species of the genus Syndesus Macleay, 1819 (Coleoptera, Lucanidae) from Ecuador. Kogane, Tokyo, 12: 1-6.

Palacios-Noé, L. K., 2016. Hongos macroscópicos del Phylum Basidiomycota en el bosque de neblina de Cuyas, Ayabaca, Piura, Perú. The Biologist (Lima), 14(2): 233256

Paulsen, M., 2010. The stag beetles of southern South America (Coleoptera: Lucanidae). Bulletin of University of Nebraska State Museum, 24: 1-148.

Pinto, J. \& Bologna, M., 1999. The New World genera of Meloidae (Coleoptera): a key and synopsis. Journal of Natural History, 33: 569-620. https://doi. org/10.1080/002229399300254

Pinto, J. \& Bologna, M., 2016. Beetles (Coleoptera) of Peru. Survey of the families. Meloidae Gyllenhal, 1810. Journal of the Kansas Entomological Society, 89(2): 202-209. https://doi.org/10.2317/JKES151211.1

Ratcliffe, B., 2015. A revised catalog of the species of Stenocrates Burmeister (Coleoptera: Scarabaeidae: Dynastinae: Cyclocephalini), with descriptions of three new species from Peru and Brazil and Stenocrates inpai Ratcliffe, 1978 placed in junior synonymy with Stenocrates popei Endrödi, 1971. The Coleopterists Bulletin, 69(4): 773-779. https://doi.org/10.1649/0010-065X-69.4.773

Ratcliffe, B., Cave, R. \& Cano, E., 2013. The Dynastinae scarab beetles of Mexico, Guatemala, and Belize (Coleoptera: Scarabaeidae: Dynastinae). Bulletin of the University of Nebraska State Museum, 27: 1-666.

Ratcliffe, B., Jameson, M., Figueroa, L., Cave, R., Paulsen, M., Cano, E., Beza-Beza, C., Jiménez-Ferbans, L. \& Reyes-Castillo, P., 2015. Beetles (Coleoptera) of Peru: a survey of the families. Scarabaeoidea. Journal of the Kansas Entomological Society, 88(2): 186-207. https:// doi.org/10.2317/kent-88-02-186-207.1

Ratcliffe, B. \& Le Tirant, S., 2017. A new species of Golofa Hope, 1837 (Coleoptera: Scarabaeidae: Dynastinae) from Peru. The Coleopterists Bulletin, 71(4): 781-785. https://doi.org/10.1649/0010-065X-71.4.781

Ratcliffe, B. C., 1976. A revision of the genus Strategus (Coleoptera: Scarabaeidae). Bulletin of the University of Nebraska State Museum, 10(3): 93-204.

Saavedra, D., Vaz de Mello, F., Ugaz, A. \& Pacherre, C., 2017. Coleópteros (Coleoptera: Scarabaeidae) de los 
bosques de niebla, Ramos y Chin Chin, Ayabaca-Huancabamba, Piura-Perú. INDES, 3(1): 108-116.

Saavedra, O., 2010. Diversidad de coleópteros (Coleoptera: Scarabaeidae) de los bosques nublados de Ramos y ChinChin, Ayabaca, Piura-Perú. Tesis para optar el título de biólogo. Universidad Nacional de Piura.

Sanabria-García, R., 2012. Estudio faunístico de la tribu Oryctini (Coleoptera: Scarabaeidae: Dynastinae) de Colombia. Tesis para optar título de Magister en Ciencias en Biología. Universidad Nacional de Colombia.

Santos-Silva, A., Komiya, Z. \& Nearns, E., 2013. Revision of the genus Prionacalus White, 1845 (Coleoptera: Cerambycidae: Prioninae: Prionini). The Coleopterists Bulletin, 67(3): 201-240. https://doi.org/10.1649/0010065X-67.3.201

Scherer, G., 1983. Diagnostic key for the Neotropical Alticine Genera (Coleoptera: Chrysomelidae: Alticinae). Entomologische Arbeiten aus dem Museum G. Frey, 31: 1-89.

Shaughney, J. \& Ratcliffe, B., 2015. A monographic revision of the genus Hoplopyga Thomson, 1880 (Coleoptera: Scarabaeidae: Cetoniinae: Gymnetini). The Coleopterists Bulletin, 69(4): 579-638. https://doi. org/10.1649/0010-065X-69.4.579

Smith, A., 2003. A monographic revision of the genus Platycoelia (Coleoptera: Scarabaeidae: Rutelinae: Anoplognathini). Bulletin of the University of Nebraska State Museum, 15: 1-202.

Smith, A. \& Skelley, P., 2007. A review of the Aphodiinae (Coleoptera: Scarabaeidae) of southern South America. Zootaxa, 1458: 1-80. https://doi.org/10.11646/zootaxa.1458.1.1

Solís, A., 2004. Métodos y técnicas de recolecta para coleópteros Scarabaeoideos. Disponible en http://unsm-ento.unl.edu/Escarabajos.htm [consultado 28 Oct. 2020].

Solís, A. \& Kohlmann, B., 2013. El género Uroxys (Coleoptera: Scarabaeidae) en Costa Rica. Giornale Italiano di Entomologia, 13(58): 289-340.
Soula, M., 1999. Rutelini 2. Revision des Anticheirina 1. Les Coleopteres du Monde. Sciences Nat. Venette, France. 116 pp.

Soula, M., 2002. Rutelini 2. Revision of the Anticheirina 2. Les Coléoptères du Monde, 26(2): 117-296.

Soula, M., 2008. Les Coléoptères du Nouveau Monde. Volume 2: Rutelini 2. Révision des Pelidnotina 2. Révision des genres: Parhoplognathus, Chipita n. gen., Heteropelidnota, Homothermon, Hoplopelidnota, Mesomerodon, Mecopelidnota, Patatra n. gen. (Coleoptera: Scarabaeidae, Rutelinae, Rutelini). Besoiro: Supplément au Bulletin de liaison de l'Association Entomologique pour la Connaissance de la Faune Tropicale. AECFT, Saintry, $40 \mathrm{pp}$.

Vaz de Mello, F., Edmonds, W., Ocampo, C. \& Schoolmeesters, P., 2011. A multilingual key to the genera and subgenera of the subfamily (Coleoptera: Scarabaeidae). Zootaxa, 2854: 1-73. https://doi.org/10.11646/ zootaxa.2854.1.1

Villarreal, H., Álvarez, M., Córdoba, F., Fagua, G., Gast, F., Mendoza, H., Ospina, M. \& Umaña, A., 2004. Manual de métodos para el desarrollo de inventarios de biodiversidad. Programa de Inventarios de Biodiversidad. Instituto de Investigación de Recursos Biológicos Alexander Von Humboldt. Bogotá. 238 pp.

Wells, S., 2007. Revision of the neotropical click beetle genus Semiotus Eschscholtz (Coleoptera: Elateridae). Contributions in Science, Natural History Museum of Los Angeles County, 514: 1-114.

Zaragoza-Caballero, S., López-Pérez, S., Vega-Badillo, V., Domínguez-León, D., Rodríguez-Mirón, G., GonzálezRamírez, M., Gutiérrez-Carranza, M., Cifuentes-Ruiz, P. \& Zurita-García, M., 2020. Luciérnagas del centro de México (Coleoptera: Lampyridae): descripción de 37 especies nuevas. Revista Mexicana de Biodiversidad, 91: 1-70. https://doi.org/10.22201/ ib.20078706e.2020.91.3104

Zelada, W. 2004. Las mariposas diurnas (Lepidoptera: Hesperioidea y Papilionoidea) del Bosque de Cuyas, Ayabaca, Piura, Perú. Revista Peruana de Entomología, 44: 37-41. 